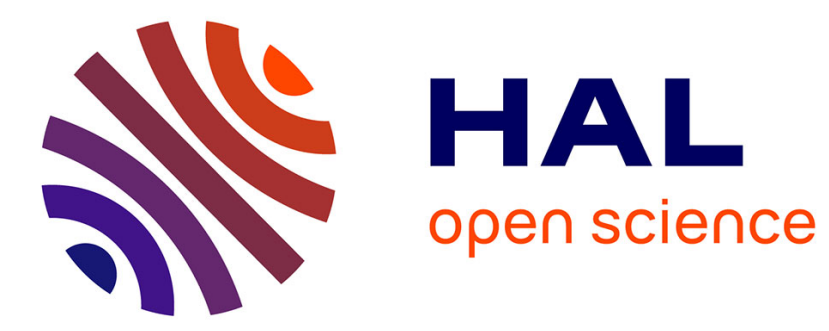

\title{
14 N Pulsed nuclear quadrupole resonance. 3. Effect of a pulse train. Optimal conditions for data averaging.
}

Maude Ferrari, Nicolas Hiblot, Alain Retournard, Daniel Canet

\section{To cite this version:}

Maude Ferrari, Nicolas Hiblot, Alain Retournard, Daniel Canet. 14 N Pulsed nuclear quadrupole resonance. 3. Effect of a pulse train. Optimal conditions for data averaging.. Molecular Physics, 2007, 105 (23-24), pp.3005-3012. 10.1080/00268970701730088 . hal-00513151

\section{HAL Id: hal-00513151 \\ https://hal.science/hal-00513151}

Submitted on 1 Sep 2010

HAL is a multi-disciplinary open access archive for the deposit and dissemination of scientific research documents, whether they are published or not. The documents may come from teaching and research institutions in France or abroad, or from public or private research centers.
L'archive ouverte pluridisciplinaire HAL, est destinée au dépôt et à la diffusion de documents scientifiques de niveau recherche, publiés ou non, émanant des établissements d'enseignement et de recherche français ou étrangers, des laboratoires publics ou privés. 


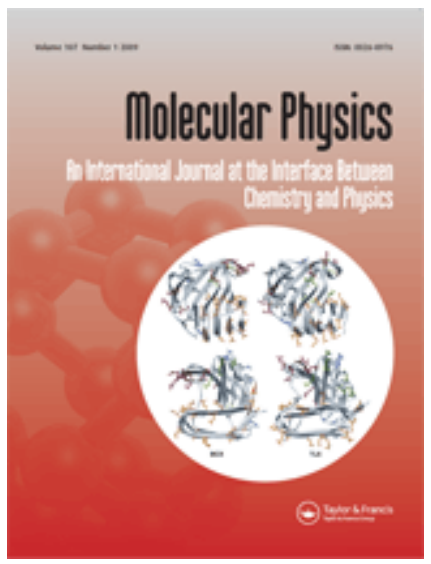

${ }^{14} \mathbf{N}$ Pulsed nuclear quadrupole resonance. 3. Effect of a pulse train. Optimal conditions for data averaging.

\begin{tabular}{|c|c|}
\hline Journal: & Molecular Physics \\
\hline Manuscript ID: & TMPH-2007-0072.R2 \\
\hline Manuscript Type: & Full Paper \\
\hline $\begin{array}{r}\text { Date Submitted by the } \\
\text { Author: }\end{array}$ & 31-Aug-2007 \\
\hline Complete List of Authors: & $\begin{array}{l}\text { CANET, Daniel; Nancy-Université, Méthodologie RMN } \\
\text { Ferrari, Maude; Nancy-Université, Méthodologie RMN } \\
\text { Hiblot, Nicolas; Nancy-Université, Méthodologie RMN } \\
\text { Retournard, Alain; Nancy-Université, Méthodologie RMN }\end{array}$ \\
\hline Keywords: & $\begin{array}{l}\text { Nuclear Quadrupole Resonance, Nitrogen-14, pulse train, data } \\
\text { averaging }\end{array}$ \\
\hline
\end{tabular}

\section{S ScholaroNE \\ Manuscript Central}




\title{
${ }^{14} \mathrm{~N}$ Pulsed nuclear quadrupole resonance. 3 . Effect of a pulse train. Optimal conditions for data averaging.
}

\author{
MAUDE FERRARI, NICOLAS HIBLOT, ALAIN RETOURNARD and DANIEL \\ CANET* \\ Méthodologie RMN (UMR CNRS-UHP 7565), Nancy-Université, Université Henri \\ Poincaré, B.P. 239 54506-Vandouvre-lès-Nancy (cedex), France \\ *Corresponding author. E-mail : Daniel.Canet@rmn.uhp-nancy.fr
}

\begin{abstract}
The calculations developed in this paper aim at determining the optimal conditions of a NQR experiment when a transition is monitored by means of a pulse train with pulses of identical duration and signal acquisition after each pulse; coherences are assumed to vanish by effective transverse relaxation prior to every new pulse. These calculations demonstrate that, as in NMR, a steady state is effectively reached for any value of the recycle time. However, by contrast with NMR, it is shown that, for optimal data averaging under steady state condition, the recycle time $T$ can be kept as low as possible (the only limitation is the acquisition time). Nutation curves (signal amplitude vs. pulse length) calculated in the steady state case are shown to depend strongly on the ratio $T / T_{1}$ ( $T_{1}$ : longitudinal relaxation time). The signal growth as a function of $T / T_{1}$ under averaging of the first transients has been evaluated as well as the number of pulses necessary for reaching a steady state.
\end{abstract}

Keywords: Nuclear Quadrupole Resonance; Nitrogen-14; pulse train; data averaging 


\section{Introduction}

Sensitivity is presumably the major problem with ${ }^{14} \mathrm{~N}$ Nuclear Quadrupole Resonance (NQR). For NMR, this can be alleviated by increasing the static magnetic field. As far as NQR is concerned, this is evidently not a solution since the resonance frequency is solely determined by the nuclear quadrupole moment and the electric field gradient (efg) at the considered nucleus. This can become dramatic for Nitrogen-14 since its resonances lie in a low frequency range $(0.7 \mathrm{MHz}-6 \mathrm{MHz})$ with especially unfavorable Botlzmann factors. When dealing with pulse experiments, the natural way of circumventing the sensitivity problem is obviously averaging. This has been considered as one of the major advantages of Fourier transform NMR and very early [1], optimal experimental conditions were determined as a function of the ratio $T / T_{1}$ where $T$ is the recycle time between two consecutive pulses and $T_{1}$ the longitudinal (spin-lattice) relaxation time. This led to the so-called Ernst angle $\cos \alpha_{\text {opt }}=T / T_{1}, \alpha_{\text {opt }}$ being the optimal flip angle related to the pulse length $\delta$ by $\alpha=\omega_{1} \delta$ with $\omega_{1}=\gamma B_{1} ; B_{1}$ : amplitude of the radio-frequency $(r f)$ field ; $\gamma:$ gyromagnetic ratio. It can be noticed that the above formula holds if and only if any transverse magnetization has disappeared prior to each new pulse by transverse (or spin-spin) relaxation process. All these considerations cannot be directly transposed to NQR, just because there is, in that case, no proportionality between an effective flip angle and the pulse duration. This is due to the fact that there is no preferred direction as the static magnetic field $B_{0}$ in NMR and that, generally, one is dealing with a powder sample (this will be the case in this paper). One has thus to take into account the orientation of each microcrystallite with respect to the direction of the $r f$ field and, in fine, to integrate over the whole sample (over all possible orientations) [2]. As a matter of fact, these features have been revisited in two 
recent papers [3-4]. We especially focused on nutation curves which represent the NQR signal amplitude as a function of the pulse length or rather as a function of $\alpha$ (as defined above). It was found, theoretically and experimentally, that nutation curves are no longer perfect sine functions as in NMR with maximum at $\alpha=90^{\circ}, 450^{\circ} \ldots$, minimum at $\alpha=270^{\circ}, 630^{\circ} \ldots$, zero at $\alpha=0^{\circ}, 180^{\circ}, 360^{\circ} \ldots$. Rather, we were able to identify two different nutations curves which look like damped sine functions [4]. One, which corresponds to an axially symmetric field gradient tensor (and thus to a single transition) with the first maximum occurring at $\alpha=101^{\circ} 5$ and the first zero at $208^{\circ}$. The other, which applies to the three transitions observed when axial symmetry is lacking. In that case, the first maximum occurs at $119^{\circ} 5$ and the first zero at $257^{\circ}$. The apparent discrepancy between these two "universal” curves was explained and discussed in ref. [4].

The aim of this paper is to determine both experimentally and theoretically how these nutation curves are modified when, in a pulse train, the recycle time is lower than $5 T_{1}$ that is when equilibrium is not reached prior to the next pulse (assumed to be immediately followed by signal acquisition). Moreover, we take advantage of this study to define the optimal conditions of signal averaging and we shall focus in particular on the number of scans necessary for attaining a steady state. Also, we shall evaluate the signal amplitude which can be obtained with a given number of scans preceding the steady state. All this results will be expressed as a function of the ratio $T / T_{1}$.

\section{Theory}

As far as a given transition is concerned, the behavior and evolution of a spin 1 system can be accounted for by a ( 2 x 2 ) density matrix as explained in previous papers [3,4] 
or by invoking the fictitious spin1/2 operator [5]. Thus, at equilibrium, the density matrix pertaining to this transition can be written as:

$$
\sigma_{e q}=\left(\begin{array}{cc}
0 & 0 \\
0 & m_{0}
\end{array}\right)
$$

where $m_{0}$ stands for the population difference between the two energy levels connected by the considered transition. In other words $m_{0}$ represents the line intensity observed when the system is at equilibrium prior to the measurement.

We shall limit ourselves to the situation of no coherence prior to the rf pulse, that is we assume that all coherences have been destroyed by the spin-spin relaxation time $T_{2}^{*}$ which includes effects of additional line broadening such as temperature variation, dipolar interactions... As coherences are associated with the two off-diagonal elements of the density matrix, the latter, immediately before the rf pulse, has therefore the form:

$$
\sigma_{-}=\left(\begin{array}{ll}
u & 0 \\
0 & v
\end{array}\right)
$$

The rf pulse is defined by its duration $\delta$ and by the amplitude $B_{1}$ of the rf field. Moreover, we have first to consider that this pulse acts on a given microcrystallite, the orientation of which is specified by the angle $\theta$ (between the symmetry axis of the efg tensor and the direction of the rf field) in such a way that we can rely on an effective flip angle (by reference to NMR):

$$
\beta=\left(\gamma B_{1} \delta\right) \sin \theta \equiv \alpha \sin \theta
$$

Note that this expression is valid only for an efg tensor of axial symmetry. In the general case (no symmetry), two polar angles are necessary (see below).

Referring again to the fictitious spin $1 / 2$, the density matrix is transformed by the rf pulse according to: 


$$
\sigma_{+}=R^{\dagger}(\beta) \sigma_{-} R(\beta)
$$

With

$$
R(\beta)=\left(\begin{array}{cc}
\cos \frac{\beta}{2} & -i \sin \frac{\beta}{2} \\
-i \sin \frac{\beta}{2} & \cos \frac{\beta}{2}
\end{array}\right)
$$

The symbol $R^{\dagger}$ indicates the transpose complex conjugate of $R$. In fact, $R(\beta)$ in (5), stands for $\exp \left(i \mathscr{H}_{\text {eff }} \delta\right)$ (see ref. [3]). We obtain easily

$$
\sigma_{+}=\left(\begin{array}{cc}
u \cos ^{2} \frac{\beta}{2}+v \sin ^{2} \frac{\beta}{2} & \frac{i}{2} \sin \beta(u-v) \\
-\frac{i}{2} \sin \beta(u-v) & u \sin ^{2} \frac{\beta}{2}+v \cos ^{2} \frac{\beta}{2}
\end{array}\right)
$$

The above calculations are of course formally equivalent to calculations which prevail for the NMR longitudinal magnetization in the case of a spin $1 / 2$ with $m_{z} \equiv m=u-v$ (see (2) ). The difference of the two diagonal elements of (6) leads, to $(u-v) \cos \beta$ which is, in NMR, the longitudinal component after a rf pulse of flip angle $\beta$. Thus, Bloch equations for longitudinal magnetization applies here with $m_{j} \equiv u_{j}-v_{j}$, which, with our notation, is the difference of diagonal elements before the $j^{\text {th }}$ pulse in a pulse train, and one has :

$$
m_{j+1}=m_{0}+\left(m_{j} \cos \beta-m_{0}\right) e^{-T / T_{1}}
$$

$T$ being the interval between two consecutive pulses. 
In order to evaluate more easily the effect of the pulse train, let us use the following notations

$$
\begin{aligned}
& E_{1}=\exp \left(-T / T_{1}\right) \\
& m_{c}=m_{0}\left(1-E_{1}\right) \\
& k=E_{1} \cos \beta
\end{aligned}
$$

The magnetization for the considered microcrystallite prior to each pulse is thus given by

$$
\begin{aligned}
& m_{1}=m_{c}+k m_{0} \\
& m_{2}=m_{c}(1+k)+k^{2} m_{0} \\
& m_{3}=m_{c}\left(1+k+k^{2}\right)+k^{3} m_{0} \\
& \vdots \\
& m_{j}=m_{c}\left(1+k+k^{2}+\cdots+k^{j-1}\right)+k^{j} m_{0}
\end{aligned}
$$

Using the well known formula for $1+k+k^{2}+\cdots+k^{j-1}$ (geometric series), we arrive at

$$
m_{j}=m_{c} \frac{1-k^{j}}{1-k}+m_{0} k^{j}
$$

Upon reinserting the quantities defined in (8), this leads to

$$
m_{j}=m_{0}\left(1-e^{-T / T_{1}}\right) \frac{1-e^{-j T / T_{1}} \cos ^{j} \beta}{1-e^{-T / T_{1}} \cos \beta}+m_{0} e^{-j T / T_{1}} \cos ^{j} \beta
$$

\subsection{Steady state}

The quantity $e^{-j T / T_{1}} \cos ^{j} \beta$ in eq. (10) gets smaller and smaller as $j$ increases so that a steady state is reached when this quantity becomes negligible and one obtains for the steady state magnetization of the considered microcrystallite: 


$$
m_{s t}=m_{0} \frac{1-e^{-T / T_{1}}}{1-\cos \beta e^{-T / T_{1}}}
$$

Which is, by nature, independent of the index $j$ and in agreement with Ernst theory [1] . These considerations justify the hypothesis of a steady state which is seen to be effectively achieved as soon as the quantity $e^{-j T / T_{1}} \cos ^{j} \beta$ is negligible with respect to unity.

As already mentioned in the introduction and in eq. (3), we denote by $\alpha$ the flip angle usually employed in NMR

$$
\alpha=\gamma B_{1} \delta
$$

As abundantly discussed and experimentally verified in ref. [3], the rf field amplitude which must be employed in NQR is twice the one which prevails in NMR. This is because, in that latter case, one has to decompose the rf field in two identical components rotating in opposite directions ; only the component which is rotating with nuclear precession is active.

Now, we derive the effective amplitude of the steady state signal following each rf pulse

$$
S_{s t}^{\omega_{0}}(\alpha) \propto\left(1-e^{-T / T_{1}}\right) \int_{0}^{\pi} \frac{\sin (\alpha \sin \theta) \sin ^{2} \theta}{1-\cos (\alpha \sin \theta) e^{-T / T_{1}}} d \theta
$$

This is just the powder average [3] which accounts for all the microcrystallite orientations with an additional $\sin \theta$ accounting for the coil receptivity.

Equation (13) can be transposed without difficulty to the case $\eta \neq 0$ for which three lines, denoted by $\omega_{x}, \omega_{y}$ and $\omega_{z}$ [6-7], exist. In that case, however, due to the lack of axial symmetry, integration has to performed over the two polar angles, $\theta$ and $\varphi$ 


$$
\begin{aligned}
& S_{s t}^{\omega_{x}}(\alpha) \propto\left(1-e^{-T / T_{1}}\right) \int_{0}^{\pi} \int_{0}^{2 \pi} \frac{\sin (\alpha \sin \theta \cos \varphi) \sin ^{2} \theta \cos \varphi}{1-\cos (\alpha \sin \theta \cos \varphi) e^{-T / T_{1}}} d \theta d \varphi \\
& S_{s t}^{\omega_{y}}(\alpha) \propto\left(1-e^{-T / T_{1}}\right) \int_{0}^{\pi} \int_{0}^{2 \pi} \frac{\sin (\alpha \sin \theta \sin \varphi) \sin ^{2} \theta \sin \varphi}{1-\cos (\alpha \sin \theta \sin \varphi) e^{-T / T_{1}}} d \theta d \varphi \\
& S_{s t}^{\omega_{z}}(\alpha) \propto\left(1-e^{-T / T_{1}}\right) \int_{0}^{\pi} \frac{\sin (\alpha \cos \theta) \cos \theta \sin \theta}{1-\cos (\alpha \cos \theta) e^{-T / T_{1}}} d \theta
\end{aligned}
$$

For symmetry reason, it is expected that (14), (15) and (16) lead to the same result in terms of nutation curve. This has been indeed verified experimentally and by numerical integration.

\subsection{Addition of the first $n$ scans}

An easy way to check the present theory against experiment is to calculate and to measure the accumulated signal amplitude resulting from $n$ scans with $n$ varying from 1 to a number necessary for reaching the above discussed steady state. Still in the case of an axially symmetric tensor, starting from (10), we can write

$$
S_{n}^{\omega_{0}}(\alpha) \propto \int_{0}^{\pi}\left[1+\sum_{j=1}^{n-1}\left(\left(1-\exp \left(-\frac{T}{T_{1}}\right)\right) \frac{1-e^{-j T / T_{1}} \cos ^{j}(\alpha \sin \theta)}{1-e^{-T / T_{1}} \cos (\alpha \sin \theta)}+e^{-j T / T_{1}} \cos ^{j}(\alpha \sin \theta)\right)\right] \sin (\alpha \sin \theta) \sin ^{2} \theta d \theta
$$

Using the formulas of geometric series, we obtain 


$$
\begin{aligned}
S_{n}^{\omega_{0}}(\alpha) \propto & \int_{0}^{\pi} \frac{1-e^{-n T / T_{1}} \cos ^{n}(\alpha \sin \theta)}{1-e^{-T / T_{1}} \cos (\alpha \sin \theta)} \sin (\alpha \sin \theta) \sin ^{2} \theta d \theta+ \\
& \int_{0}^{\pi} \frac{1-e^{-T / T_{1}}}{1-e^{-T / T_{1}} \cos (\alpha \sin \theta)}\left(n-\frac{1-e^{-n T / T_{1}} \cos ^{n}(\alpha \sin \theta)}{1-e^{-T / T_{1}} \cos (\alpha \sin \theta)}\right) \sin (\alpha \sin \theta) \sin ^{2} \theta d \theta
\end{aligned}
$$

Again, we can transpose this expression to the case $\eta \neq 0$. Recognizing that the same result will hold for $\omega_{x}, \omega_{y}$ and $\omega_{z}$, we just quite give the equation related to $\omega_{x}$

$$
\begin{aligned}
& S_{n}^{\omega_{x}}(\alpha) \propto \int_{0}^{\pi} \int_{0}^{2 \pi} \frac{1-e^{-n T / T_{1}} \cos ^{n}(\alpha \sin \theta \cos \varphi)}{1-e^{-T / T_{1}} \cos (\alpha \sin \theta \cos \varphi)} \sin (\alpha \sin \theta \cos \varphi) \sin ^{2} \theta \cos \varphi d \theta d \varphi+ \\
& \int_{0}^{\pi} \int_{0}^{2 \pi} \frac{1-e^{-T / T_{1}}}{1-e^{-T / T_{1}} \cos (\alpha \sin \theta \cos \varphi)}\left(n-\frac{1-e^{-n T / T_{1}} \cos ^{n}(\alpha \sin \theta \cos \varphi)}{1-e^{-T / T_{1}} \cos (\alpha \sin \theta \cos \varphi)}\right) \sin (\alpha \sin \theta \cos \varphi) \sin ^{2} \theta \cos \varphi d \theta d \varphi
\end{aligned}
$$

\section{Simulations and experimental verifications}

Experiments have been carried out with a prototype spectrometer entirely built in this laboratory [8]. The probe coil is a solenoid of $4.5 \mathrm{~cm}$ height and of $13 \mathrm{~mm}$ diameter. The powder is contained in a standard $10 \mathrm{~mm}$ o.d. NMR tube filled approximately at 5 cm height. For sensitivity reasons, the theory developed in the previous section has only been verified with sodium nitrite for two of its three lines (three lines because $\eta \neq 0$ ) at 4.6 $\mathrm{MHz}\left(\omega_{x}\right)$ and $3.6 \mathrm{MHz}\left(\omega_{y}\right)$. The probe containing the sample is placed in a insulated box for avoiding spurious effects due to temperature fluctuations. The output power of the amplifier has been limited to $100 \mathrm{~W}$, which corresponds to a pulse length of about $10 \mu$ s for the first maximum (pseudo $90^{\circ}$ pulse). The $B_{1}$ value was experimentally determined from the pulse length corresponding to the first maximum of the nutation curve obtained in equilibrium conditions. 
The $T_{1}$ value at $4.6 \mathrm{MHz}$ and $3.6 \mathrm{MHz}$ are respectively $70 \mathrm{~ms}$ and $200 \mathrm{~ms}$ [9]. Simulations based on formulas (13)-(14) on the one hand, and (18)-(19) on the other hand, have been performed by numerical integration according to procedures previously published [3].

In figure 1, are shown simulated nutation curves corresponding to the steady state for different values of the ratio $T / T_{1}$. Having in mind the comparison with sodium nitrite experimental data, these calculations were derived from formula (14). The same trends show up for the case $\eta=0$. In any case, the displayed experimental data are obtained after a number of dummy scans derived from the curves of figure 2 to make sure that a steady state is actually reached. As shown in figure 3 , for $\alpha$ values up to $170^{\circ}$, the agreement between experiments and simulations is generally very good, especially for the position of the first maximum. However, presumably for instrumental reasons (possible droop of the amplifier, inhomogeneity of the $B_{1}$ field), there is a deviation at relatively high pulse lengths. The position of the first maximum is displayed in figure 4 as a function of the ratio $T / T_{1}$. It is seen from theory and experiment that this position decreases when the ratio $T / T_{1}$ decreases. By contrast, the first zero always occurs at the same value of $\alpha$. This can be qualitatively understood by comparison with NMR. Of course, the major differences between NMR and NQR lie in the position of singularities (maximums, zeros, minimums) and in the damping of NQR nutation curves, the powder average being responsible for these features. Let us go back nevertheless to NMR for which the signal (under steady state conditions) is given by

$$
S_{N M R}(\alpha)=\frac{\sin \alpha\left(1-e^{-T / T_{1}}\right)}{1-\cos \alpha \mathrm{e}^{-T / T_{1}}}
$$


It is well known that the first maximum corresponds to a flip angle value which is the Ernst angle (see above): $\cos \alpha_{\text {opt }}=T / T_{1}$. This demonstrates that the position of the first maximum decreases when $T / T_{1}$ also decreases. Moreover, from the above formula, it can be seen that the first zero is obtained for $\sin \alpha=0$, thus for $\alpha=\pi$, independent of $T / T_{1}$. These two properties are indeed retrieved, mutatis mutandis, in the NQR nutation curves.

In a practical prospect, the next issue to be addressed is the choice of the optimal ratio $T / T_{1}$ for a given measuring time. Assuming data averaging, this is obtained simply by multiplying eqs.(13) and (14-16) by $\sqrt{T_{1} / T}$. Results, deduced from simulations are plotted in figure 5 where, for each ratio $T / T_{1}$, the $\alpha$ value leading to the first maximum (pseudo $90^{\circ}$ pulse) has been chosen for these simulations. Surprisingly and by contrast with NMR, there is not really a optimum. Signal intensity keeps increasing when $T / T_{1}$ decreases. Thus, in both cases $(\eta=0$ and $\eta \neq 0)$, it is recommended to choose the shortest repetition time compatible with the duration of data acquisition. This is confirmed by the experimental results shown in figure 6 .

Finally, it is interesting to determine the number of scans necessary for reaching a steady state. For that purpose, we have calculated the accumulated signal amplitude from formulas (18) and (19). Both leading to similar results, we just show those corresponding to sodium nitrite for which we possess experimental data (formula (19); $\eta \neq 0)$. We relied on the $\mathrm{NaNO}_{2}$ line at $4.6 \mathrm{MHz}$ because the signal is visible in a single scan. Signal amplitudes (simulated) presented in figure 2 have been divided by $n$ (number of scans) just to show what is the contribution of every new scan. It can be seen that smaller is the ratio $T / T_{1}$, larger is the number of scans for reaching the steady state. For instance, for $T / T_{1}=2$ the steady state is obtained after 10 scans whereas, for 
$T / T_{1}=0.3$ this requires 20 scans. These results are of some importance for the design of experiments necessitating the accumulations of a relatively large number of transients. Conversely, if, for some reasons, it is necessary to add truly identical signal, these results provide the number of scans which have to be disregarded (dummy scans, see above). These results are confirmed by experimental data reported in figure 7 according to the usual representation.

\section{Conclusion}

As recognized in our previous papers [3-4], the major differences between liquid state NMR and powder NQR arise from the necessity of integrating over the polar angles defining the direction of the rf field within each microcrystallite. In the case of NQR, nutation curves are no longer perfect sine functions with extremes occurring at multiples of $\pi / 2$. Rather, one observes sorts of damped sine functions with extremes at values generally larger than the multiple of $\pi / 2$ indicated above. This is true however when the system returns to complete equilibrium before each new rf pulse. The present paper deals with the non-equilibrium situation and several simulations, along with experimental verifications, have been performed as a function of $T / T_{1}$ ( $T$ : repetition time, $T_{1}$ : longitudinal relaxation time). It can be noticed that the relevant calculations have nothing to do with the so-called Steady State Free Precession (SSPF) method [1011], this one implying a first pseudo $90^{\circ}$ pulse followed by a train of pseudo $180^{\circ}$ pulses and thus concerning coherences. Rather, the present study is concerned with populations (the equivalent of the NMR longitudinal magnetization) and implies that coherences vanish before every new pulse. We have shown that, as expected, a steady state is reached but we have calculated the number of scans (as a function of the ratio $T / T_{1}$ ) 
required for arriving at this steady state. This number of scans increases dramatically when $T / T_{1}$ gets smaller than unity. Under steady state conditions, nutation curves evolve as a function of $T / T_{1}$ and the first maximum occurs at a value which decreases when $T / T_{1}$ decreases. By contrast, the first zero is quasi independent of $T / T_{1}$. Finally, we have determined the conditions for obtaining the best signal-to-noise ratio for a given measuring time (and under steady state conditions). Contrary to a naive prediction, it is the shortest $T / T_{1}$ ratio which leads to the best result. The repetition time should therefore be always kept to the minimum value enabling to acquire correctly the free induction decay (fid). Owing to the inherently poor sensitivity of nitrogen-14 NQR, these findings are of some importance if NQR would become a technique used on a routine basis.

This work is part of the ANR project "Instrumentation in Magnetic Resonance" (Grant Blan06-2_139020) 


\section{References}

[1] R.R.Ernst, Adv. Magn. Reson., 2, 1 (1966).

[2] S.Vega, J. Chem. Phys., 61, 1093 (1974).

[3] B.Cordier, D.Grandclaude, A.Retournard, L.Merlat, D.Canet, Molec. Phys., 103, 2593 (2005).

[4] D.Canet, L.Merlat, B.Cordier, D.Grandclaude, A.Retournard, M.Ferrari, Molec. Phys., 104, 1391 (2006).

[5] Y.K.Lee, Conc.Magn.Res. 14, 155 (2002).

[6] J.A.S. Smith, J. Chem. Educ. 48, 39 (1971).

[7] J.A.S. Smith, Chem.Soc.Rev. 15, 225 (1986).

[8] a) B.Cordier, PhD Thesis, Université H.Poincaré (2005).

b) A.Retournard, N.Hiblot, M.Ferrari, D.Grandclaude, D.Canet, to be published.

[9] G.Petersen, P.J.Bray, J. Chem. Phys., 64, 522, (1976).

[10] T.N.Rudakov, V.T.Mikhaltsevitch, J.H.Flexman, Sol.St. NMR, 25, 94 (2004).

[11] T.N.Rudakov, V.T.Mikhaltsevitch, J.H.Flexman, Sol.St. NMR, 25, 112 (2004). 
Figure captions

Figure 1. Theoretical nutation curves for the steady state in the case $\eta \neq 0$. From top to bottom $T / T_{1}=5, T / T_{1}=3, T / T_{1}=2, T / T_{1}=1.3, T / T_{1}=0.5$ as a function of $\alpha=\gamma B_{1} \delta$.

Figure 2. Plot of the signal amplitude obtained after the first $\mathrm{n}$ scans divided by $\mathrm{n}$ for different values of the ratio $T / T_{1}$ ( from top to bottom : 5, 3, 2, 1.3, 0.3, 0.1). These simulations are based on formula (19).

Figure 3. Theoretical nutation curves along with experimental data. Empty squares : $4.64 \mathrm{MHz}$ transition ; filled lozenges : 3.6 MHz transition.

Figure 4. Evolution of the value of the angle $\alpha\left(\alpha=\gamma B_{1} \delta\right)$ corresponding to the first maximum of the nutation curve as a function of the ratio $T / T_{1}$. Experimental data are displayed according to the symbols of figure 3 .

Figure 5. Plot of the maximum signal which can be obtained in a given measuring time, as a function of the ratio $T / T_{1}$. Full line: $\eta=0$; dashed line: $\eta \neq 0$.

Figure 6. 4.6MHz spectra (resulting from the addition of time domain data) obtained in 22s for different values of the ratio $T / T_{1}$; from top to bottom: 1.3, 2, 3, 5 with the following experimental parameters (ns : number of scans)

\begin{tabular}{|c|c|c|}
\hline$T / T_{1}$ & $\mathrm{~ns}$ & $\mathrm{~T}(\mathrm{~ms})$ \\
\hline 1.3 & 224 & 90 \\
2 & 157 & 140 \\
3 & 105 & 210 \\
5 & 63 & 350 \\
\hline
\end{tabular}


Figure 7. Plot of the signal amplitude obtained after the first n scans (formula (19)) for the following values of the ratio $T / T_{1}: 5,3,2,1.3$ (from top to the bottom). Lozenges, triangles, circles, squares represent the corresponding experimental data of $\mathrm{NaNO}_{2}$ at $4.6 \mathrm{MHz}$. 11 12 13 14 15 16 17 18 19 20 21 22 23 24 25 26 27 28 29 30 31 32 33 34 35 36 37 38 39 40 41 42 43 44 45 46 47 48 49 50 51 52 53 54 55 56 57 58 59 60 


1
2
3
4
5
6
7
8
9
10
11
12
13
14
15
16
17
18
19
20
21
22
23
24
25
26
27
28
29
30
31
32
33
34
35
36
37
38
39
40
41
42
43
44
45
46
47
48
49
50
51
52
53
54
55
56
57
58
59
60

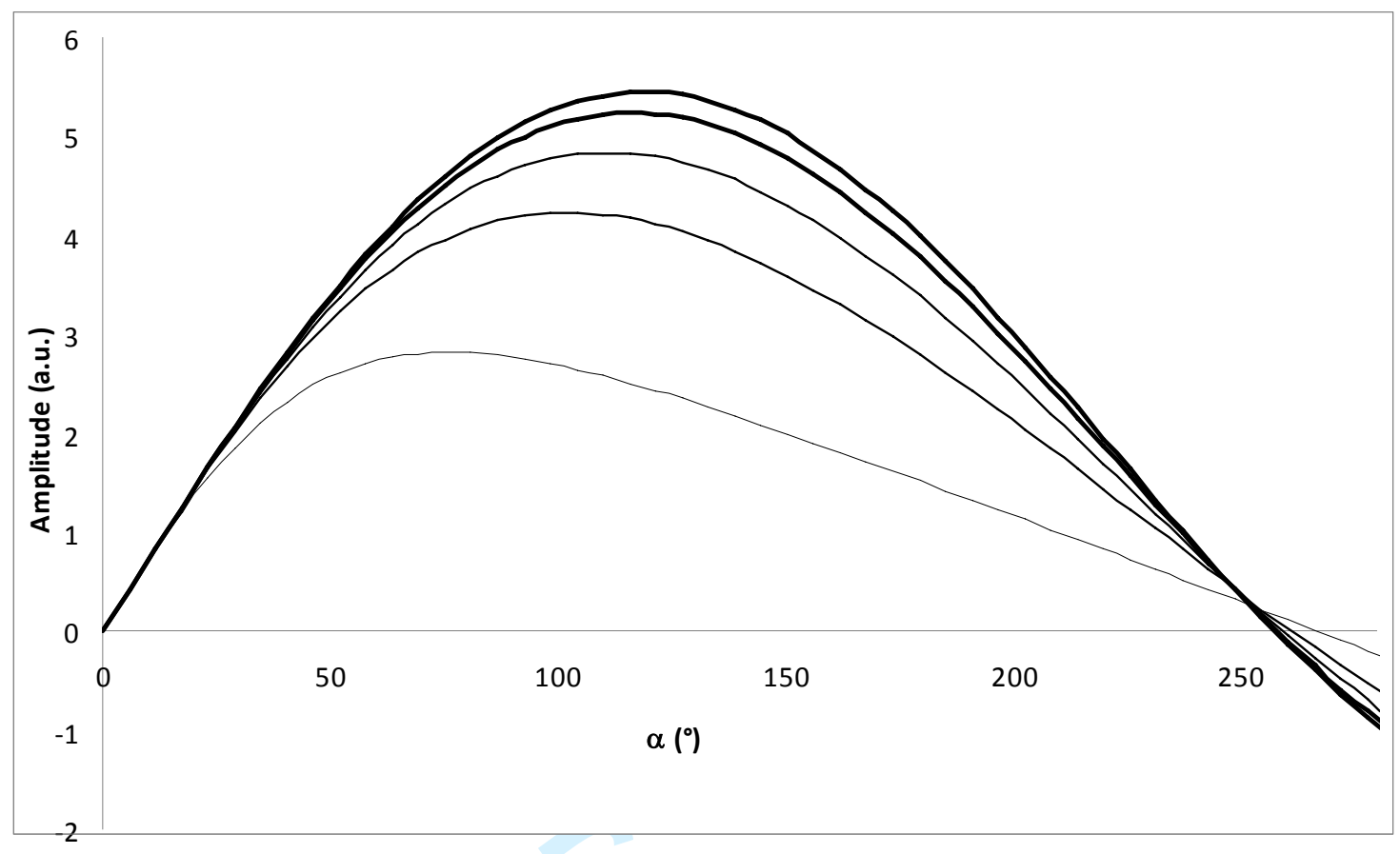

Figure 1 


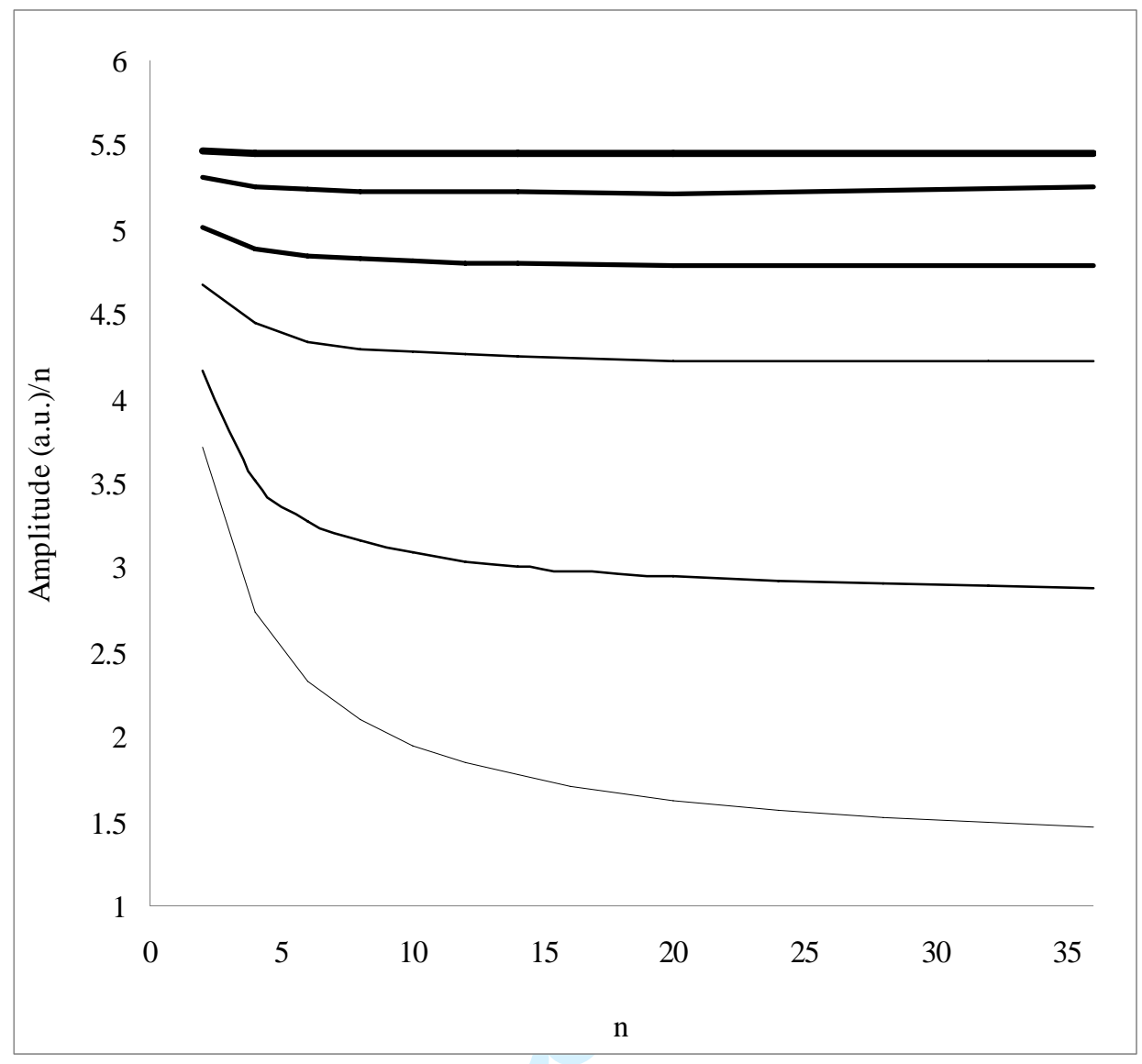

Figure 2 

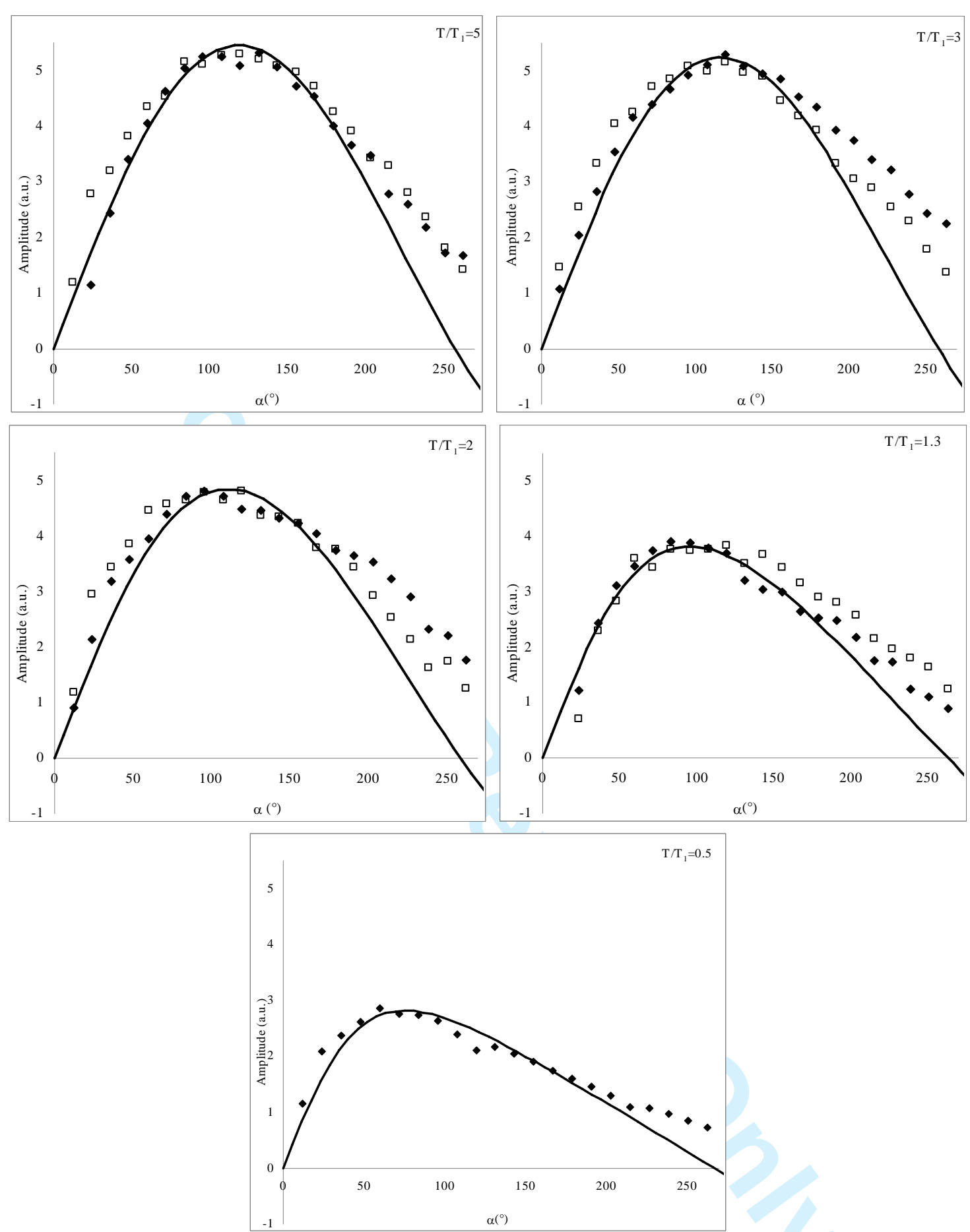

Figure 3 


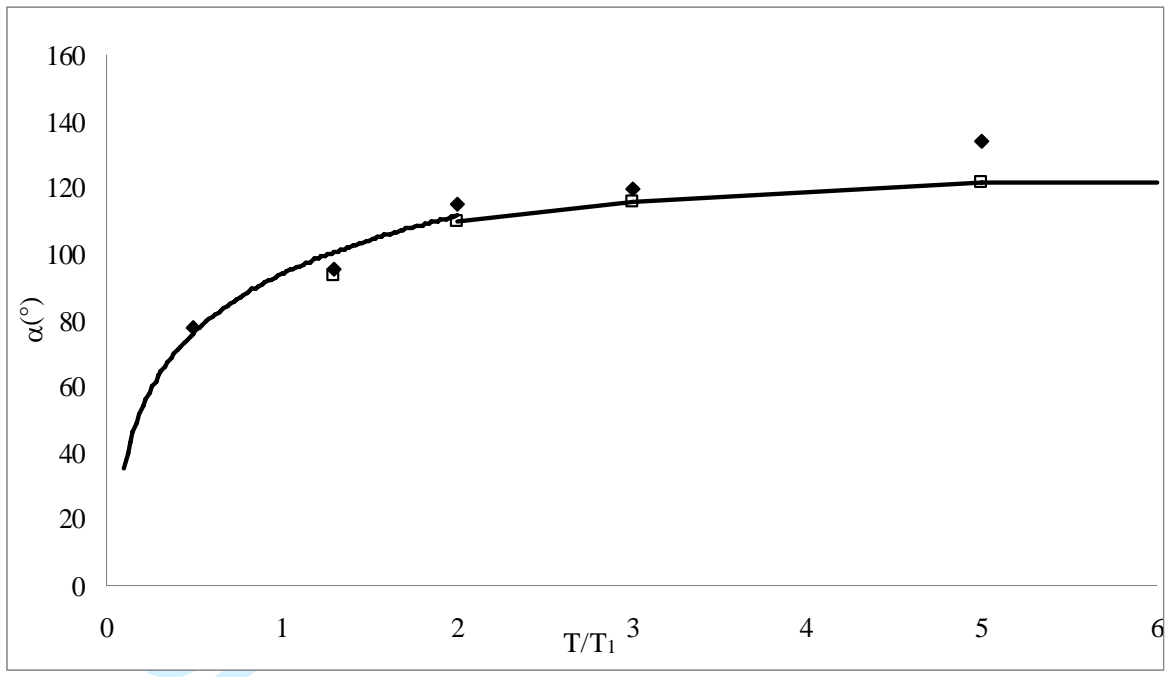

Figure 4 


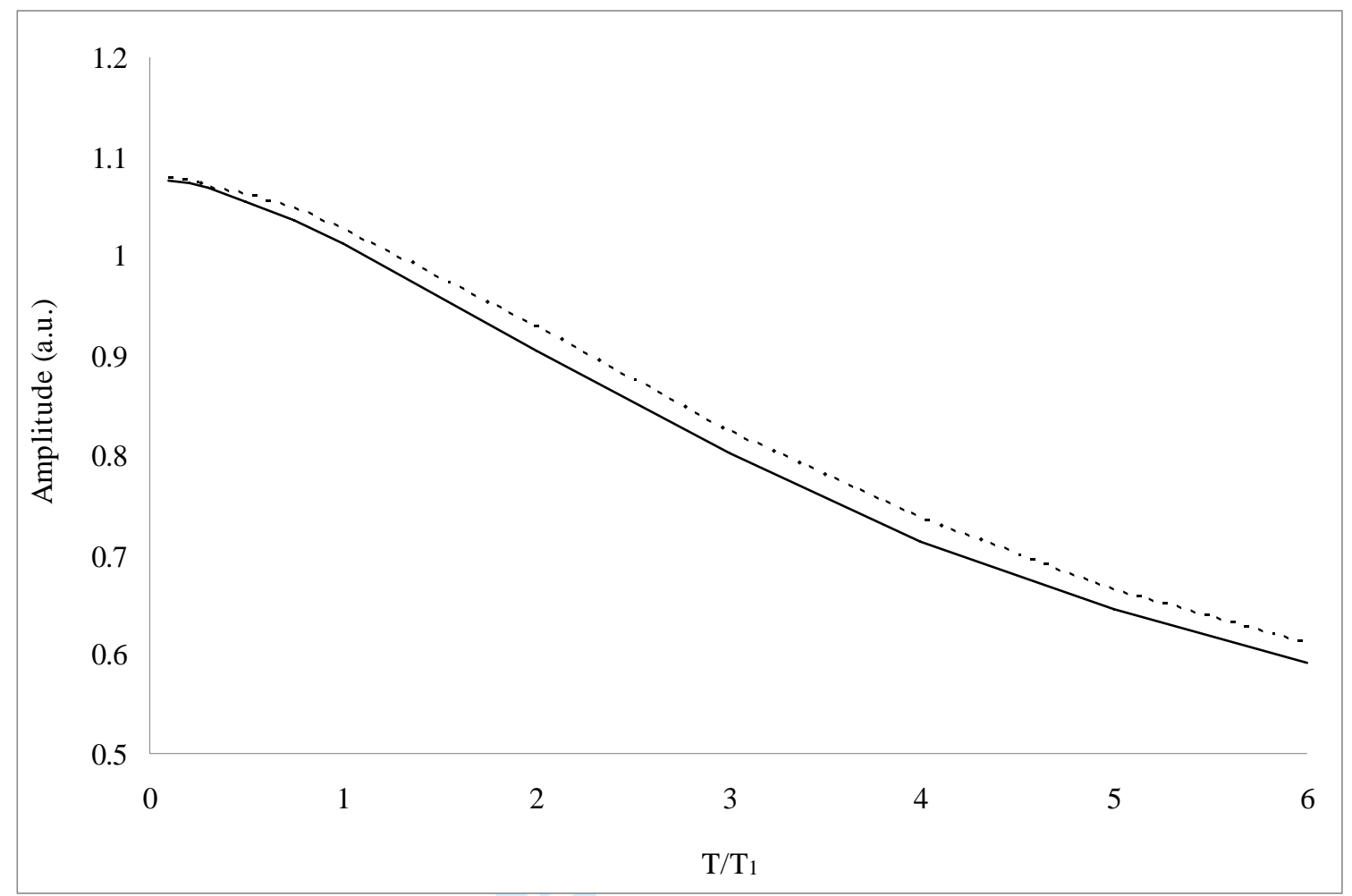

Figure 5 


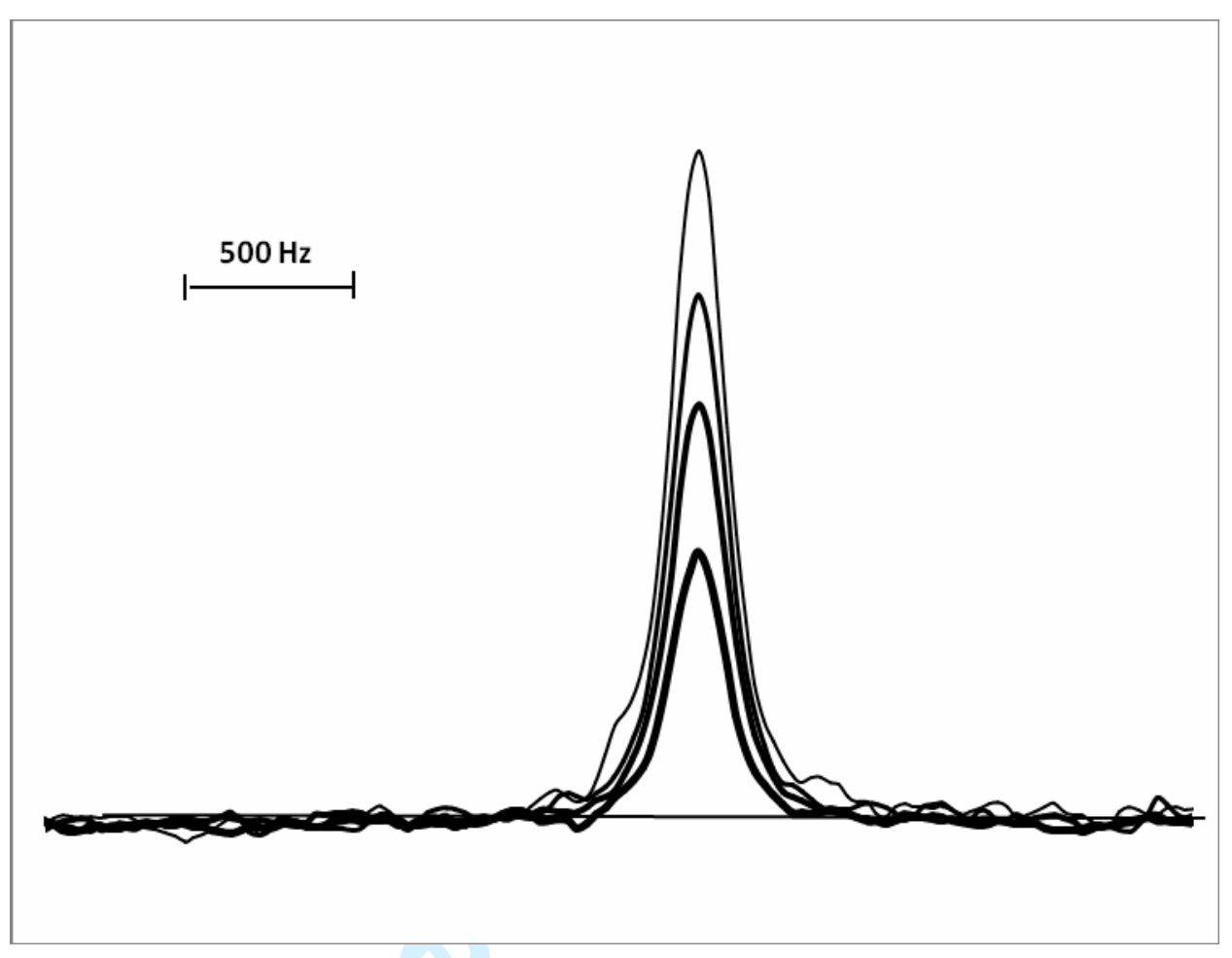

Figure 6 


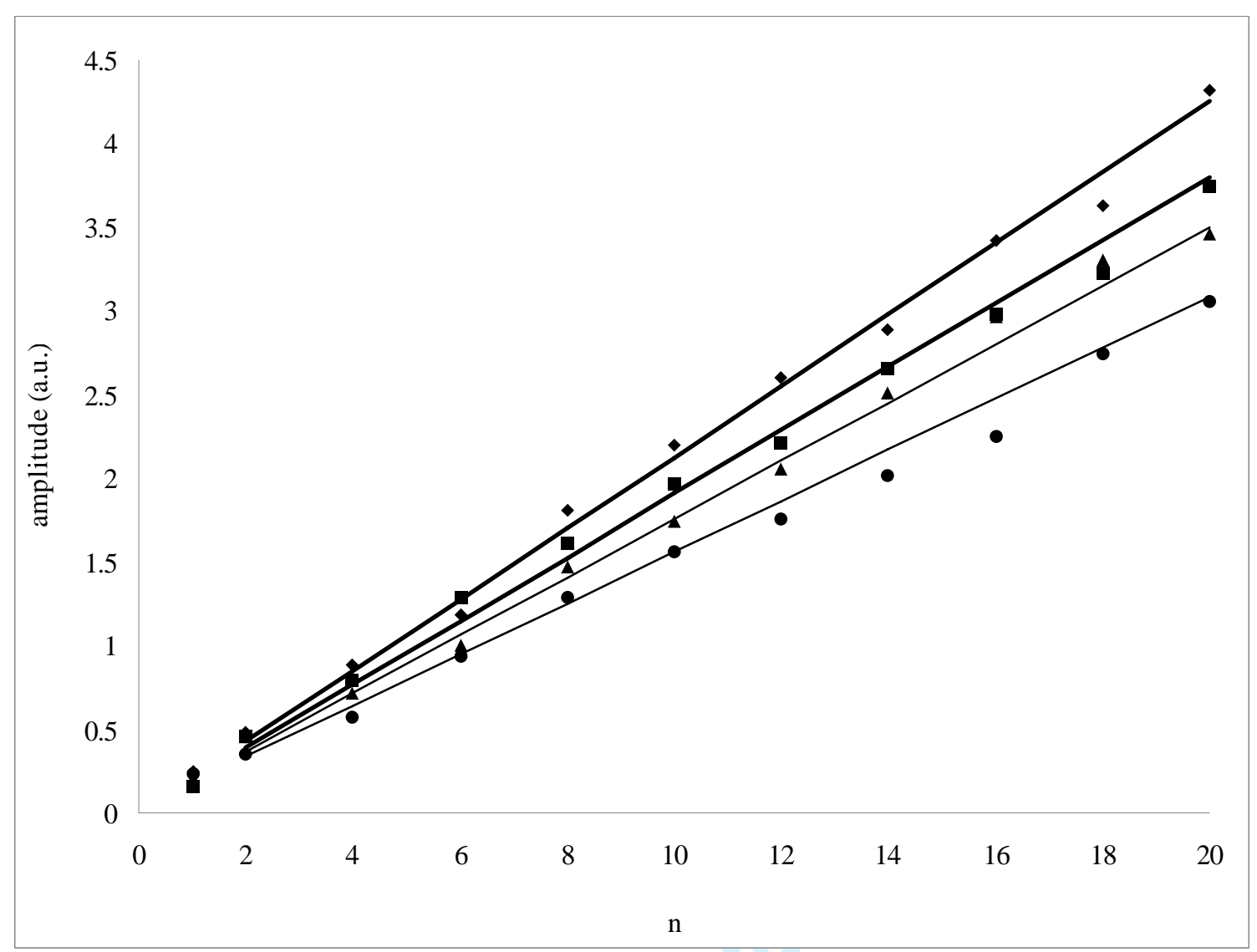

Figure 7 


\title{
${ }^{14} \mathrm{~N}$ Pulsed nuclear quadrupole resonance. 3. Effect of a pulse train. Optimal conditions for data averaging.
}

\author{
MAUDE FERRARI, NICOLAS HIBLOT, ALAIN RETOURNARD and DANIEL \\ CANET* \\ Méthodologie RMN (UMR CNRS-UHP 7565), Nancy-Université, Université Henri \\ Poincaré, B.P. 239 54506-Vandœuvre-lès-Nancy (cedex), France \\ *Corresponding author. E-mail : Daniel.Canet@rmn.uhp-nancy.fr
}

\begin{abstract}
The calculations developed in this paper aim at determining the optimal conditions of a NQR experiment when a transition is monitored by means of a pulse train with pulses of identical duration and signal acquisition after each pulse; coherences are assumed to vanish by effective transverse relaxation prior to every new pulse. These calculations demonstrate that, as in NMR, a steady state is effectively reached for any value of the recycle time. However, by contrast with NMR, it is shown that, for optimal data averaging under steady state condition, the recycle time $T$ can be kept as low as possible (the only limitation is the acquisition time). Nutation curves (signal amplitude vs. pulse length) calculated in the steady state case are shown to depend strongly on the ratio $T / T_{1}$ ( $T_{1}$ : longitudinal relaxation time). The signal growth as a function of $T / T_{1}$ under averaging of the first transients has been evaluated as well as the number of pulses necessary for reaching a steady state.
\end{abstract}

Keywords: Nuclear Quadrupole Resonance; Nitrogen-14; pulse train; data averaging 


\section{Introduction}

Sensitivity is presumably the major problem with ${ }^{14} \mathrm{~N}$ Nuclear Quadrupole Resonance (NQR). For NMR, this can be alleviated by increasing the static magnetic field. As far as NQR is concerned, this is evidently not a solution since the resonance frequency is solely determined by the nuclear quadrupole moment and the electric field gradient (efg) at the considered nucleus. This can become dramatic for Nitrogen-14 since its resonances lie in a low frequency range $(0.7 \mathrm{MHz}-6 \mathrm{MHz})$ with especially unfavorable Botlzmann factors. When dealing with pulse experiments, the natural way of circumventing the sensitivity problem is obviously averaging. This has been considered as one of the major advantages of Fourier transform NMR and very early [1], optimal experimental conditions were determined as a function of the ratio $T / T_{1}$ where $T$ is the recycle time between two consecutive pulses and $T_{1}$ the longitudinal (spin-lattice) relaxation time. This led to the so-called Ernst angle $\cos \alpha_{\text {opt }}=T / T_{1}, \alpha_{\text {opt }}$ being the optimal flip angle related to the pulse length $\delta$ by $\alpha=\omega_{1} \delta$ with $\omega_{1}=\gamma B_{1} ; B_{1}$ : amplitude of the radio-frequency $(r f)$ field ; $\gamma:$ gyromagnetic ratio. It can be noticed that the above formula holds if and only if any transverse magnetization has disappeared prior to each new pulse by transverse (or spin-spin) relaxation process. All these considerations cannot be directly transposed to NQR, just because there is, in that case, no proportionality between an effective flip angle and the pulse duration. This is due to the fact that there is no preferred direction as the static magnetic field $B_{0}$ in NMR and that, generally, one is dealing with a powder sample (this will be the case in this paper). One has thus to take into account the orientation of each microcrystallite with respect to the direction of the $r f$ field and, in fine, to integrate over the whole sample (over all possible orientations) [2]. As a matter of fact, these features have been revisited in two 
recent papers [3-4]. We especially focused on nutation curves which represent the NQR signal amplitude as a function of the pulse length or rather as a function of $\alpha$ (as defined above). It was found, theoretically and experimentally, that nutation curves are no longer perfect sine functions as in NMR with maximum at $\alpha=90^{\circ}, 450^{\circ} \ldots$, minimum at $\alpha=270^{\circ}, 630^{\circ} \ldots$, zero at $\alpha=0^{\circ}, 180^{\circ}, 360^{\circ} \ldots$. Rather, we were able to identify two different nutations curves which look like damped sine functions [4]. One, which corresponds to an axially symmetric field gradient tensor (and thus to a single transition) with the first maximum occurring at $\alpha=101^{\circ} 5$ and the first zero at $208^{\circ}$. The other, which applies to the three transitions observed when axial symmetry is lacking. In that case, the first maximum occurs at $119^{\circ} 5$ and the first zero at $257^{\circ}$. The apparent discrepancy between these two "universal" curves was explained and discussed in ref. [4].

The aim of this paper is to determine both experimentally and theoretically how these nutation curves are modified when, in a pulse train, the recycle time is lower than $5 T_{1}$ that is when equilibrium is not reached prior to the next pulse (assumed to be immediately followed by signal acquisition). Moreover, we take advantage of this study to define the optimal conditions of signal averaging and we shall focus in particular on the number of scans necessary for attaining a steady state. Also, we shall evaluate the signal amplitude which can be obtained with a given number of scans preceding the steady state. All this results will be expressed as a function of the ratio $T / T_{1}$.

\section{Theory}

As far as a given transition is concerned, the behavior and evolution of a spin 1 system can be accounted for by a ( 2 × 2 ) density matrix as explained in previous papers [3,4] 
or by invoking the fictitious spin $1 / 2$ operator [5]. Thus, at equilibrium, the density matrix pertaining to this transition can be written as:

$$
\sigma_{e q}=\left(\begin{array}{cc}
0 & 0 \\
0 & m_{0}
\end{array}\right)
$$

where $m_{0}$ stands for the population difference between the two energy levels connected by the considered transition. In other words $m_{0}$ represents the line intensity observed when the system is at equilibrium prior to the measurement.

We shall limit ourselves to the situation of no coherence prior to the rf pulse, that is we assume that all coherences have been destroyed by the spin-spin relaxation time $T_{2}^{*}$ which includes effects of additional line broadening such as temperature variation, dipolar interactions... As coherences are associated with the two off-diagonal elements of the density matrix, the latter, immediately before the rf pulse, has therefore the form:

$$
\sigma_{-}=\left(\begin{array}{ll}
u & 0 \\
0 & v
\end{array}\right)
$$

The rf pulse is defined by its duration $\delta$ and by the amplitude $B_{1}$ of the rf field. Moreover, we have first to consider that this pulse acts on a given microcrystallite, the orientation of which is specified by the angle $\theta$ (between the symmetry axis of the efg tensor and the direction of the rf field) in such a way that we can rely on an effective flip angle (by reference to NMR):

$$
\beta=\left(\gamma B_{1} \delta\right) \sin \theta \equiv \alpha \sin \theta
$$

Note that this expression is valid only for an efg tensor of axial symmetry. In the general case (no symmetry), two polar angles are necessary (see below).

Referring again to the fictitious spin $1 / 2$, the density matrix is transformed by the $\mathrm{rf}$ pulse according to: 


$$
\sigma_{+}=R^{\dagger}(\beta) \sigma_{-} R(\beta)
$$

With

$$
R(\beta)=\left(\begin{array}{cc}
\cos \frac{\beta}{2} & -i \sin \frac{\beta}{2} \\
-i \sin \frac{\beta}{2} & \cos \frac{\beta}{2}
\end{array}\right)
$$

The symbol $R^{\dagger}$ indicates the transpose complex conjugate of $R$. In fact, $R(\beta)$ in (5), stands for $\exp \left(i \mathrm{H}_{e f f} \delta\right)$ (see ref. [3]). We obtain easily

$$
\sigma_{+}=\left(\begin{array}{cc}
u \cos ^{2} \frac{\beta}{2}+v \sin ^{2} \frac{\beta}{2} & \frac{i}{2} \sin \beta(u-v) \\
-\frac{i}{2} \sin \beta(u-v) & u \sin ^{2} \frac{\beta}{2}+v \cos ^{2} \frac{\beta}{2}
\end{array}\right)
$$

The above calculations are of course formally equivalent to calculations which prevail for the NMR longitudinal magnetization in the case of a spin $1 / 2$ with $m_{z} \equiv m=u-v$ (see (2) ). The difference of the two diagonal elements of (6) leads, to $(u-v) \cos \beta$ which is, in NMR, the longitudinal component after a rf pulse of flip angle $\beta$. Thus, Bloch equations for longitudinal magnetization applies here with $m_{j} \equiv u_{j}-v_{j}$, which, with our notation, is the difference of diagonal elements before the $j^{\text {th }}$ pulse in a pulse train, and one has :

$$
m_{j+1}=m_{0}+\left(m_{j} \cos \beta-m_{0}\right) e^{-T / T_{1}}
$$

$T$ being the interval between two consecutive pulses. 
In order to evaluate more easily the effect of the pulse train, let us use the following notations

$$
\begin{aligned}
& E_{1}=\exp \left(-T / T_{1}\right) \\
& m_{c}=m_{0}\left(1-E_{1}\right) \\
& k=E_{1} \cos \beta
\end{aligned}
$$

The magnetization for the considered microcrystallite prior to each pulse is thus given by

$$
\begin{aligned}
& m_{1}=m_{c}+k m_{0} \\
& m_{2}=m_{c}(1+k)+k^{2} m_{0} \\
& m_{3}=m_{c}\left(1+k+k^{2}\right)+k^{3} m_{0} \\
& \vdots \\
& m_{j}=m_{c}\left(1+k+k^{2}+\cdots+k^{j-1}\right)+k^{j} m_{0}
\end{aligned}
$$

Using the well known formula for $1+k+k^{2}+\cdots+k^{j-1}$ (geometric series), we arrive at

$$
m_{j}=m_{c} \frac{1-k^{j}}{1-k}+m_{0} k^{j}
$$

Upon reinserting the quantities defined in (8), this leads to

$$
m_{j}=m_{0}\left(1-e^{-T / T_{1}}\right) \frac{1-e^{-j T / T_{1}} \cos ^{j} \beta}{1-e^{-T / T_{1}} \cos \beta}+m_{0} e^{-j T / T_{1}} \cos ^{j} \beta
$$

\subsection{Steady state}

The quantity $e^{-j T / T_{1}} \cos ^{j} \beta$ in eq. (10) gets smaller and smaller as $j$ increases so that a steady state is reached when this quantity becomes negligible and one obtains for the steady state magnetization of the considered microcrystallite: 


$$
m_{s t}=m_{0} \frac{1-e^{-T / T_{1}}}{1-\cos \beta e^{-T / T_{1}}}
$$

Which is, by nature, independent of the index $j$ and in agreement with Ernst theory [1] . These considerations justify the hypothesis of a steady state which is seen to be effectively achieved as soon as the quantity $e^{-j T / T_{1}} \cos ^{j} \beta$ is negligible with respect to unity.

As already mentioned in the introduction and in eq. (3), we denote by $\alpha$ the flip angle usually employed in NMR

$$
\alpha=\gamma B_{1} \delta
$$

As abundantly discussed and experimentally verified in ref. [3], the rf field amplitude which must be employed in NQR is twice the one which prevails in NMR. This is because, in that latter case, one has to decompose the rf field in two identical components rotating in opposite directions ; only the component which is rotating with nuclear precession is active.

Now, we derive the effective amplitude of the steady state signal following each rf pulse

$$
S_{s t}^{\omega_{0}}(\alpha) \propto\left(1-e^{-T / T_{1}}\right) \int_{0}^{\pi} \frac{\sin (\alpha \sin \theta) \sin ^{2} \theta}{1-\cos (\alpha \sin \theta) e^{-T / T_{1}}} d \theta
$$

This is just the powder average [3] which accounts for all the microcrystallite orientations with an additional $\sin \theta$ accounting for the coil receptivity.

Equation (13) can be transposed without difficulty to the case $\eta \neq 0$ for which three lines, denoted by $\omega_{x}, \omega_{y}$ and $\omega_{z}$ [6-7], exist. In that case, however, due to the lack of axial symmetry, integration has to performed over the two polar angles, $\theta$ and $\varphi$ 


$$
\begin{aligned}
& S_{s t}^{\omega_{x}}(\alpha) \propto\left(1-e^{-T / T_{1}}\right) \int_{0}^{\pi} \int_{0}^{2 \pi} \frac{\sin (\alpha \sin \theta \cos \varphi) \sin ^{2} \theta \cos \varphi}{1-\cos (\alpha \sin \theta \cos \varphi) e^{-T / T_{1}}} d \theta d \varphi \\
& S_{s t}^{\omega_{y}}(\alpha) \propto\left(1-e^{-T / T_{1}}\right) \int_{0}^{\pi} \int_{0}^{2 \pi} \frac{\sin (\alpha \sin \theta \sin \varphi) \sin ^{2} \theta \sin \varphi}{1-\cos (\alpha \sin \theta \sin \varphi) e^{-T / T_{1}}} d \theta d \varphi \\
& S_{s t}^{\omega_{z}}(\alpha) \propto\left(1-e^{-T / T_{1}}\right) \int_{0}^{\pi} \frac{\sin (\alpha \cos \theta) \cos \theta \sin \theta}{1-\cos (\alpha \cos \theta) e^{-T / T_{1}}} d \theta
\end{aligned}
$$

For symmetry reason, it is expected that (14), (15) and (16) lead to the same result in terms of nutation curve. This has been indeed verified experimentally and by numerical integration.

\subsection{Addition of the first $n$ scans}

An easy way to check the present theory against experiment is to calculate and to measure the accumulated signal amplitude resulting from $n$ scans with $n$ varying from 1 to a number necessary for reaching the above discussed steady state. Still in the case of an axially symmetric tensor, starting from (10), we can write

$$
S_{n}^{\omega_{0}}(\alpha) \propto \int_{0}^{\pi}\left[1+\sum_{j=1}^{n-1}\left(\left(1-\exp \left(-\frac{T}{T_{1}}\right)\right) \frac{1-e^{-j T / T_{1}} \cos ^{j}(\alpha \sin \theta)}{1-e^{-T / T_{1}} \cos (\alpha \sin \theta)}+e^{-j T / T_{1}} \cos ^{j}(\alpha \sin \theta)\right)\right] \sin (\alpha \sin \theta) \sin ^{2} \theta d \theta
$$

Using the formulas of geometric series, we obtain 


$$
\begin{aligned}
S_{n}^{\omega_{0}}(\alpha) \propto & \int_{0}^{\pi} \frac{1-e^{-n T / T_{1}} \cos ^{n}(\alpha \sin \theta)}{1-e^{-T / T_{1}} \cos (\alpha \sin \theta)} \sin (\alpha \sin \theta) \sin ^{2} \theta d \theta+ \\
& \int_{0}^{\pi} \frac{1-e^{-T / T_{1}}}{1-e^{-T / T_{1}} \cos (\alpha \sin \theta)}\left(n-\frac{1-e^{-n T / T_{1}} \cos ^{n}(\alpha \sin \theta)}{1-e^{-T / T_{1}} \cos (\alpha \sin \theta)}\right) \sin (\alpha \sin \theta) \sin ^{2} \theta d \theta
\end{aligned}
$$

Again, we can transpose this expression to the case $\eta \neq 0$. Recognizing that the same result will hold for $\omega_{x}, \omega_{y}$ and $\omega_{z}$, we just quite give the equation related to $\omega_{x}$

$$
\begin{aligned}
& S_{n}^{\omega_{x}}(\alpha) \propto \int_{0}^{\pi} \int_{0}^{2 \pi} \frac{1-e^{-n T / T_{1}} \cos ^{n}(\alpha \sin \theta \cos \varphi)}{1-e^{-T / T_{1}} \cos (\alpha \sin \theta \cos \varphi)} \sin (\alpha \sin \theta \cos \varphi) \sin ^{2} \theta \cos \varphi d \theta d \varphi+ \\
& \int_{0}^{\pi} \int_{0}^{2 \pi} \frac{1-e^{-T / T_{1}}}{1-e^{-T / T_{1}} \cos (\alpha \sin \theta \cos \varphi)}\left(n-\frac{1-e^{-n T / T_{1}} \cos ^{n}(\alpha \sin \theta \cos \varphi)}{1-e^{-T / T_{1}} \cos (\alpha \sin \theta \cos \varphi)}\right) \sin (\alpha \sin \theta \cos \varphi) \sin ^{2} \theta \cos \varphi d \theta d \varphi
\end{aligned}
$$

\section{Simulations and experimental verifications}

Experiments have been carried out with a prototype spectrometer entirely built in this laboratory [8]. The probe coil is a solenoid of $4.5 \mathrm{~cm}$ height and of $13 \mathrm{~mm}$ diameter. The powder is contained in a standard $10 \mathrm{~mm}$ o.d. NMR tube filled approximately at 5 $\mathrm{cm}$ height. For sensitivity reasons, the theory developed in the previous section has only been verified with sodium nitrite for two of its three lines (three lines because $\eta \neq 0$ ) at 4.6 $\mathrm{MHz}\left(\omega_{x}\right)$ and $3.6 \mathrm{MHz}\left(\omega_{y}\right)$. The probe containing the sample is placed in a insulated box for avoiding spurious effects due to temperature fluctuations. The output power of the amplifier has been limited to $100 \mathrm{~W}$, which corresponds to a pulse length of about $10 \mu \mathrm{s}$ for the first maximum (pseudo $90^{\circ}$ pulse). The $B_{1}$ value was experimentally determined from the pulse length corresponding to the first maximum of the nutation curve obtained in equilibrium conditions. 
The $T_{1}$ value at $4.6 \mathrm{MHz}$ and $3.6 \mathrm{MHz}$ are respectively $70 \mathrm{~ms}$ and $200 \mathrm{~ms}$ [9]. Simulations based on formulas (13)-(14) on the one hand, and (18)-(19) on the other hand, have been performed by numerical integration according to procedures previously published [3].

In figure 1, are shown simulated nutation curves corresponding to the steady state for different values of the ratio $T / T_{1}$. Having in mind the comparison with sodium nitrite experimental data, these calculations were derived from formula (14). The same trends show up for the case $\eta=0$. In any case, the displayed experimental data are obtained after a number of dummy scans derived from the curves of figure 2 to make sure that a steady state is actually reached. As shown in figure 3 , for $\alpha$ values up to $170^{\circ}$, the agreement between experiments and simulations is generally very good, especially for the position of the first maximum. However, presumably for instrumental reasons (possible droop of the amplifier, inhomogeneity of the $B_{1}$ field), there is a deviation at relatively high pulse lengths. The position of the first maximum is displayed in figure 4 as a function of the ratio $T / T_{1}$. It is seen from theory and experiment that this position decreases when the ratio $T / T_{1}$ decreases. By contrast, the first zero always occurs at the same value of $\alpha$. This can be qualitatively understood by comparison with NMR. Of course, the major differences between NMR and NQR lie in the position of singularities (maximums, zeros, minimums) and in the damping of NQR nutation curves, the powder average being responsible for these features. Let us go back nevertheless to NMR for which the signal (under steady state conditions) is given by

$$
S_{N M R}(\alpha)=\frac{\sin \alpha\left(1-e^{-T / T_{1}}\right)}{1-\cos \alpha \mathrm{e}^{-T / T_{1}}}
$$


It is well known that the first maximum corresponds to a flip angle value which is the Ernst angle (see above): $\cos \alpha_{\text {opt }}=T / T_{1}$. This demonstrates that the position of the first maximum decreases when $T / T_{1}$ also decreases. Moreover, from the above formula, it can be seen that the first zero is obtained for $\sin \alpha=0$, thus for $\alpha=\pi$, independent of $T / T_{1}$. These two properties are indeed retrieved, mutatis mutandis, in the NQR nutation curves.

In a practical prospect, the next issue to be addressed is the choice of the optimal ratio $T / T_{1}$ for a given measuring time. Assuming data averaging, this is obtained simply by multiplying eqs.(13) and (14-16) by $\sqrt{T_{1} / T}$. Results, deduced from simulations are plotted in figure 5 where, for each ratio $T / T_{1}$, the $\alpha$ value leading to the first maximum (pseudo $90^{\circ}$ pulse) has been chosen for these simulations. Surprisingly and by contrast with NMR, there is not really a optimum. Signal intensity keeps increasing when $T / T_{1}$ decreases. Thus, in both cases $(\eta=0$ and $\eta \neq 0)$, it is recommended to choose the shortest repetition time compatible with the duration of data acquisition. This is confirmed by the experimental results shown in figure 6 .

Finally, it is interesting to determine the number of scans necessary for reaching a steady state. For that purpose, we have calculated the accumulated signal amplitude from formulas (18) and (19). Both leading to similar results, we just show those corresponding to sodium nitrite for which we possess experimental data (formula (19); $\eta \neq 0)$. We relied on the $\mathrm{NaNO}_{2}$ line at $4.6 \mathrm{MHz}$ because the signal is visible in a single scan. Signal amplitudes (simulated) presented in figure 2 have been divided by $n$ (number of scans) just to show what is the contribution of every new scan. It can be seen that smaller is the ratio $T / T_{1}$, larger is the number of scans for reaching the steady state. For instance, for $T / T_{1}=2$ the steady state is obtained after 10 scans whereas, for 


\section{Conclusion}

As recognized in our previous papers [3-4], the major differences between liquid state NMR and powder NQR arise from the necessity of integrating over the polar angles defining the direction of the rf field within each microcrystallite. In the case of NQR, nutation curves are no longer perfect sine functions with extremes occurring at multiples of $\pi / 2$. Rather, one observes sorts of damped sine functions with extremes at values generally larger than the multiple of $\pi / 2$ indicated above. This is true however when the system returns to complete equilibrium before each new rf pulse. The present paper deals with the non-equilibrium situation and several simulations, along with experimental verifications, have been performed as a function of $T / T_{1}$ ( $T:$ repetition time, $T_{1}$ : longitudinal relaxation time). It can be noticed that the relevant calculations have nothing to do with the so-called Steady State Free Precession (SSPF) method [1011], this one implying a first pseudo $90^{\circ}$ pulse followed by a train of pseudo $180^{\circ}$ pulses and thus concerning coherences. Rather, the present study is concerned with populations (the equivalent of the NMR longitudinal magnetization) and implies that coherences vanish before every new pulse. We have shown that, as expected, a steady state is reached but we have calculated the number of scans (as a function of the ratio $T / T_{1}$ ) 
required for arriving at this steady state. This number of scans increases dramatically when $T / T_{1}$ gets smaller than unity. Under steady state conditions, nutation curves evolve as a function of $T / T_{1}$ and the first maximum occurs at a value which decreases when $T / T_{1}$ decreases. By contrast, the first zero is quasi independent of $T / T_{1}$. Finally, we have determined the conditions for obtaining the best signal-to-noise ratio for a given measuring time (and under steady state conditions). Contrary to a naive prediction, it is the shortest $T / T_{1}$ ratio which leads to the best result. The repetition time should therefore be always kept to the minimum value enabling to acquire correctly the free induction decay (fid). Owing to the inherently poor sensitivity of nitrogen-14 NQR, these findings are of some importance if NQR would become a technique used on a routine basis.

This work is part of the ANR project "Instrumentation in Magnetic Resonance" (Grant Blan06-2_139020) 


\section{References}

[1] R.R.Ernst, Adv. Magn. Reson., 2, 1 (1966).

[2] S.Vega, J. Chem. Phys., 61, 1093 (1974).

[3] B.Cordier, D.Grandclaude, A.Retournard, L.Merlat, D.Canet, Molec. Phys., 103, 2593 (2005).

[4] D.Canet, L.Merlat, B.Cordier, D.Grandclaude, A.Retournard, M.Ferrari, Molec. Phys., 104, 1391 (2006).

[5] Y.K.Lee, Conc.Magn.Res. 14, 155 (2002).

[6] J.A.S. Smith, J. Chem. Educ. 48, 39 (1971).

[7] J.A.S. Smith, Chem.Soc.Rev. 15, 225 (1986).

[8] a) B.Cordier, PhD Thesis, Université H.Poincaré (2005).

b) A.Retournard, N.Hiblot, M.Ferrari, D.Grandclaude, D.Canet, to be published.

[9] G.Petersen, P.J.Bray, J. Chem. Phys., 64, 522, (1976).

[10] T.N.Rudakov, V.T.Mikhaltsevitch, J.H.Flexman, Sol.St. NMR, 25, 94 (2004).

[11] T.N.Rudakov, V.T.Mikhaltsevitch, J.H.Flexman, Sol.St. NMR, 25, 112 (2004). 
Figure captions

Figure 1. Theoretical nutation curves for the steady state in the case $\eta \neq 0$. From top to bottom $T / T_{1}=5, T / T_{1}=3, T / T_{1}=2, T / T_{1}=1.3, T / T_{1}=0.5$ as a function of $\alpha=\gamma B_{1} \delta$.

Figure 2. Plot of the signal amplitude obtained after the first $\mathrm{n}$ scans divided by $\mathrm{n}$ for different values of the ratio $T / T_{1}$ ( from top to bottom : $5,3,2,1.3,0.3,0.1$ ). These simulations are based on formula (19).

Figure 3. Theoretical nutation curves along with experimental data. Empty squares : $4.64 \mathrm{MHz}$ transition ; filled lozenges : $3.6 \mathrm{MHz}$ transition.

Figure 4. Evolution of the value of the angle $\alpha\left(\alpha=\gamma B_{1} \delta\right)$ corresponding to the first maximum of the nutation curve as a function of the ratio $T / T_{1}$. Experimental data are displayed according to the symbols of figure 3.

Figure 5. Plot of the maximum signal which can be obtained in a given measuring time, as a function of the ratio $T / T_{1}$. Full line: $\eta=0$; dashed line: $\eta \neq 0$.

Figure 6. 4.6MHz spectra (resulting from the addition of time domain data) obtained in $22 \mathrm{~s}$ for different values of the ratio $T / T_{1}$; from top to bottom: $1.3,2,3,5$ with the following experimental parameters (ns : number of scans)

\begin{tabular}{|c|c|c|}
\hline$T / T_{1}$ & $\mathrm{~ns}$ & $\mathrm{~T}(\mathrm{~ms})$ \\
\hline 1.3 & 224 & 90 \\
2 & 157 & 140 \\
3 & 105 & 210 \\
5 & 63 & 350 \\
\hline
\end{tabular}


Figure 7. Plot of the signal amplitude obtained after the first $\mathrm{n}$ scans (formula (19)) for the following values of the ratio $T / T_{1}: 5,3,2,1.3$ (from top to the bottom). Lozenges, triangles, circles, squares represent the corresponding experimental data of $\mathrm{NaNO}_{2}$ at $4.6 \mathrm{MHz}$. 


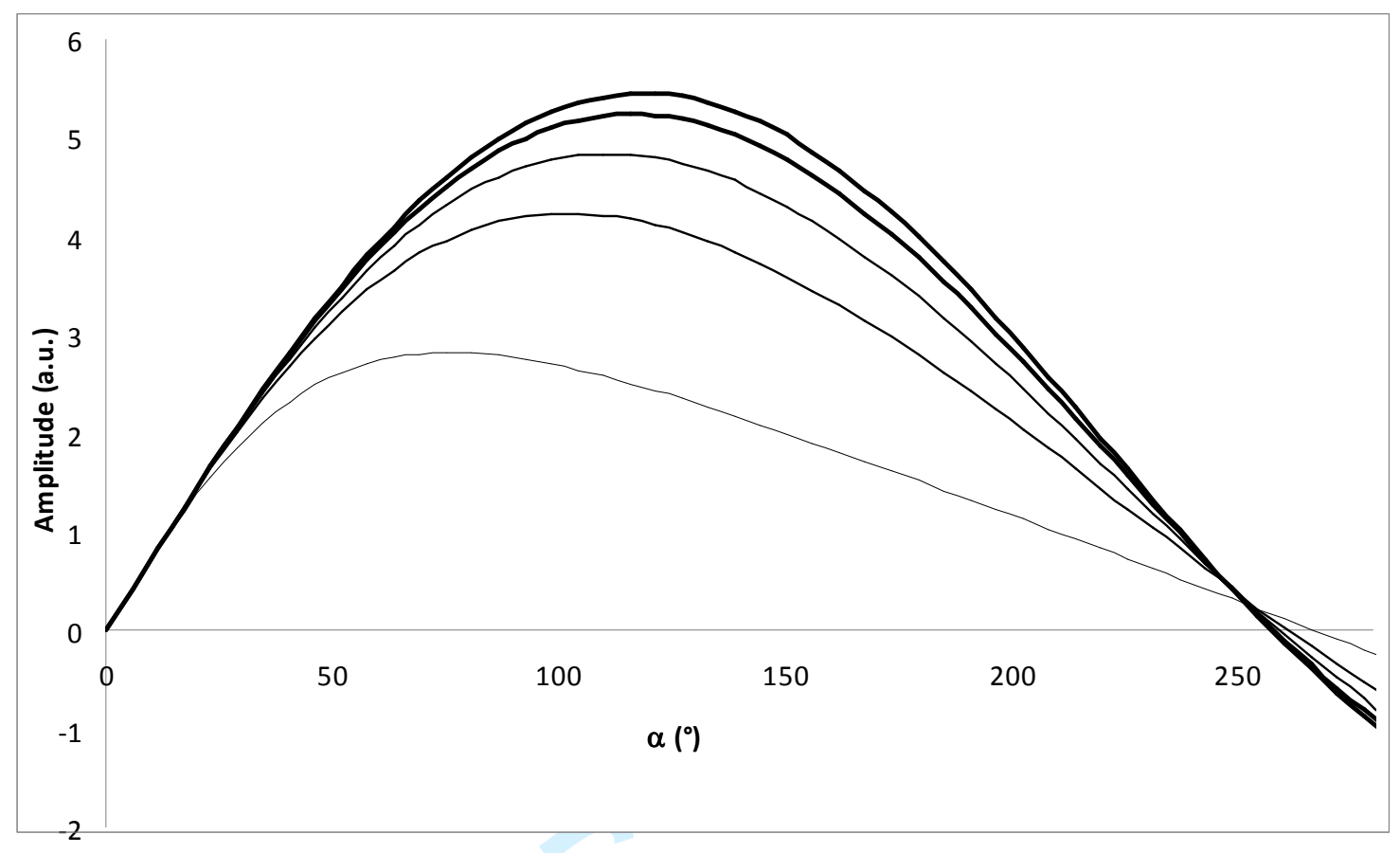

Figure 1 


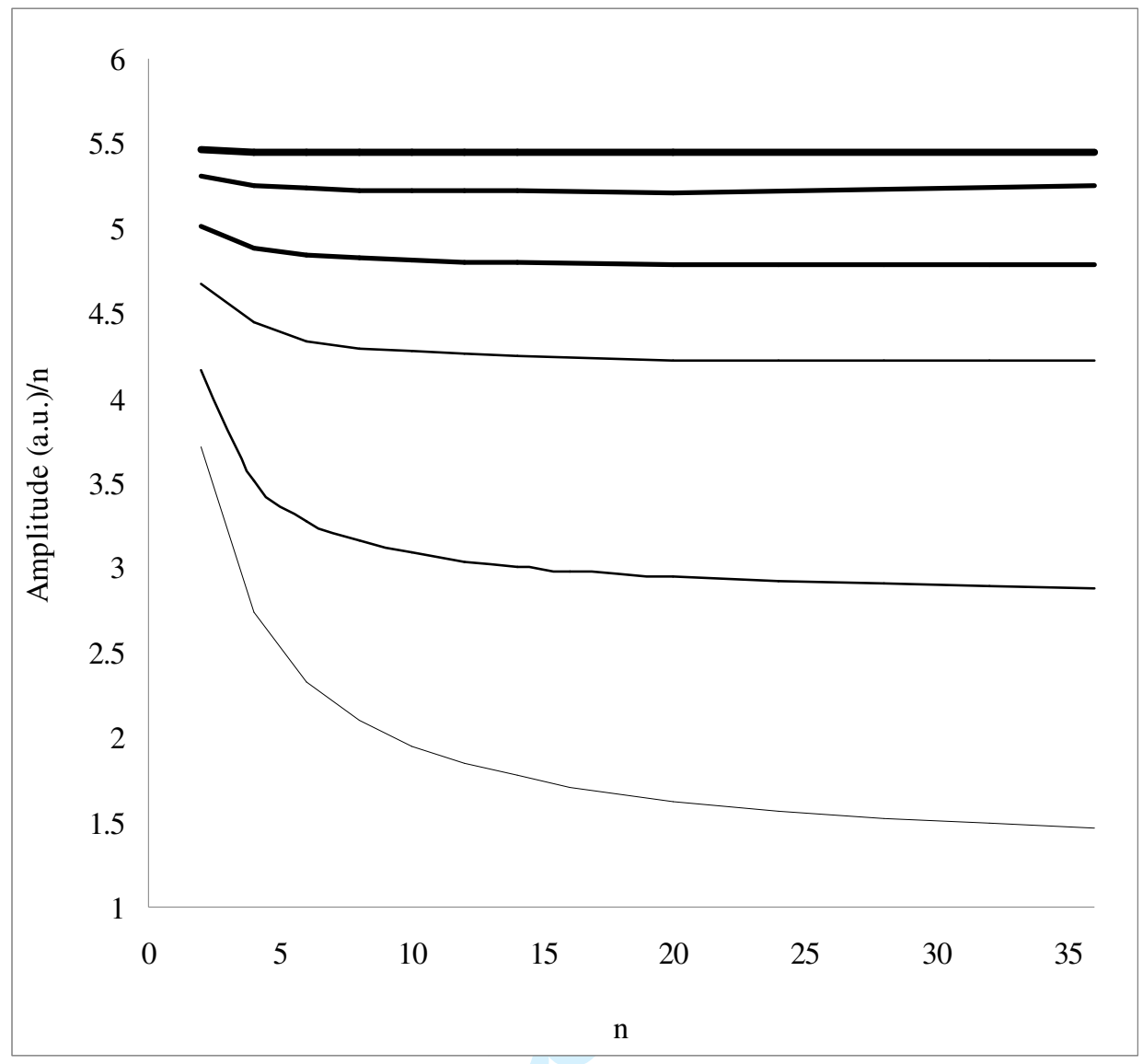

Figure 2 

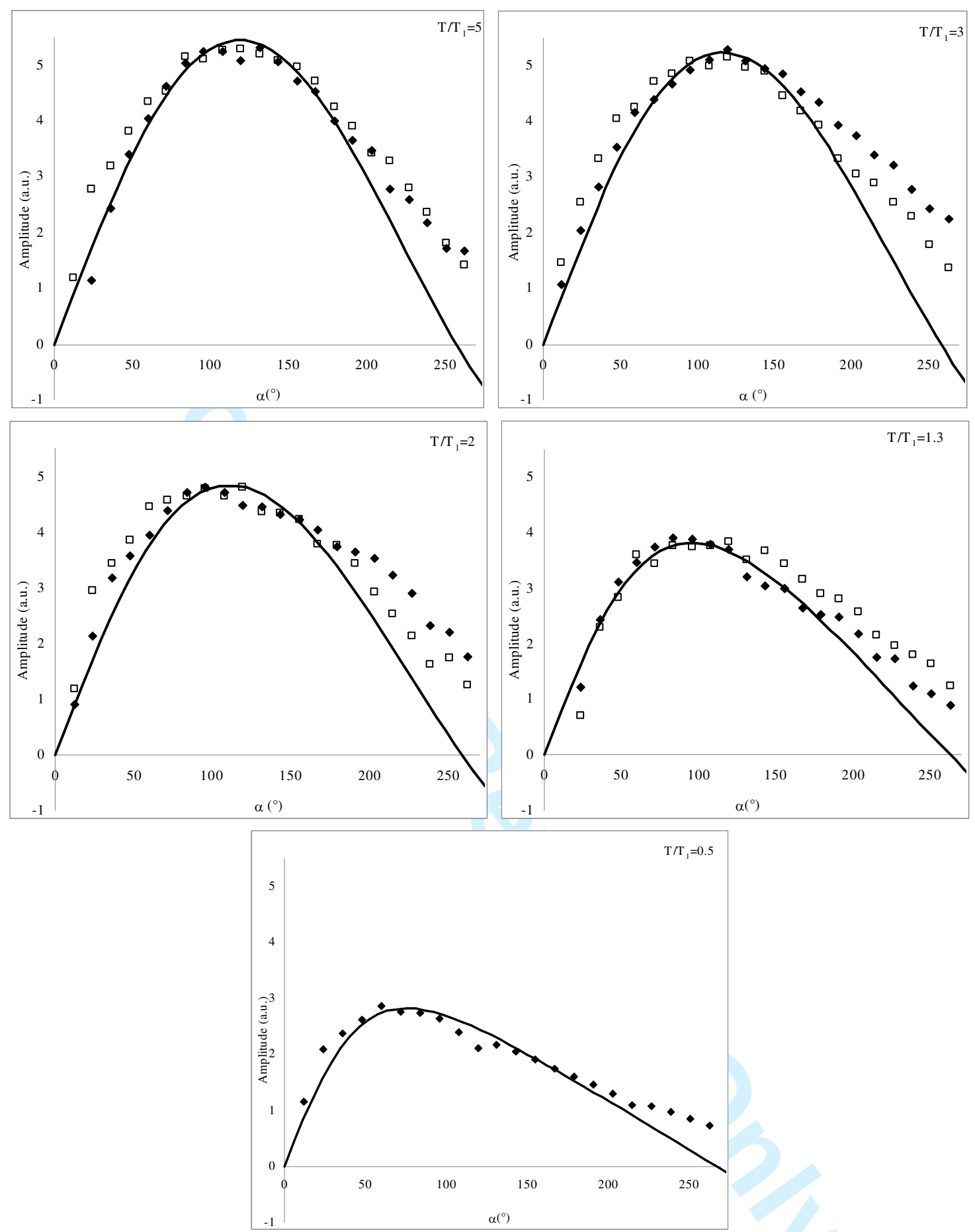

Figure 3 


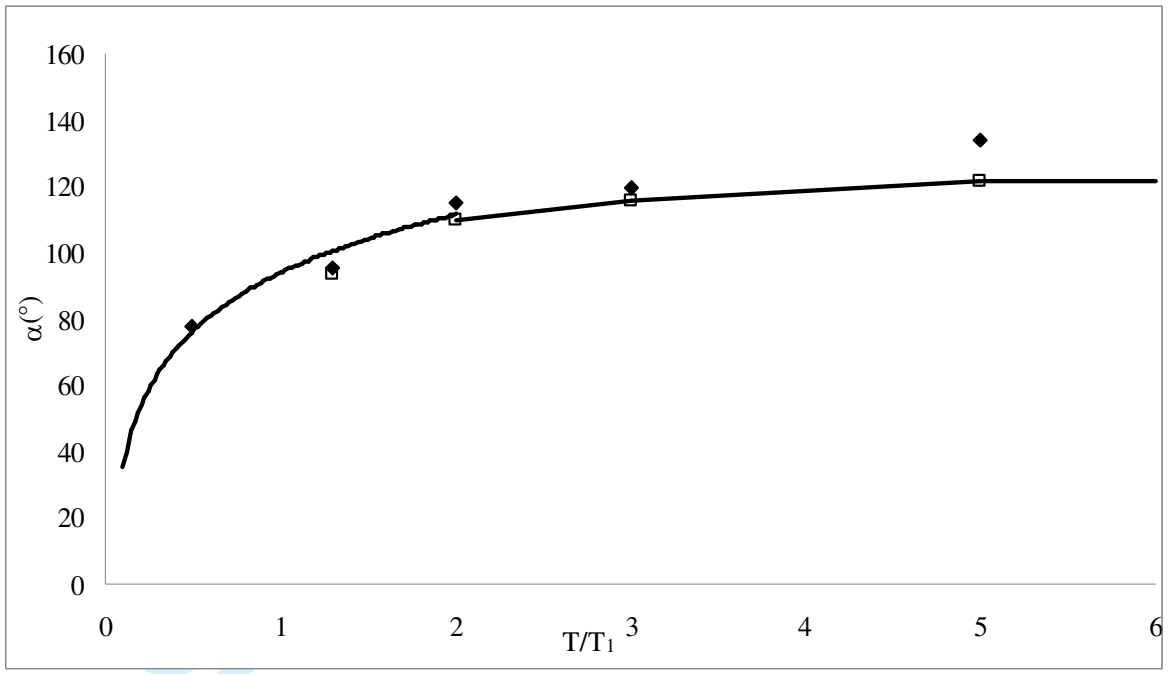

Figure 4 


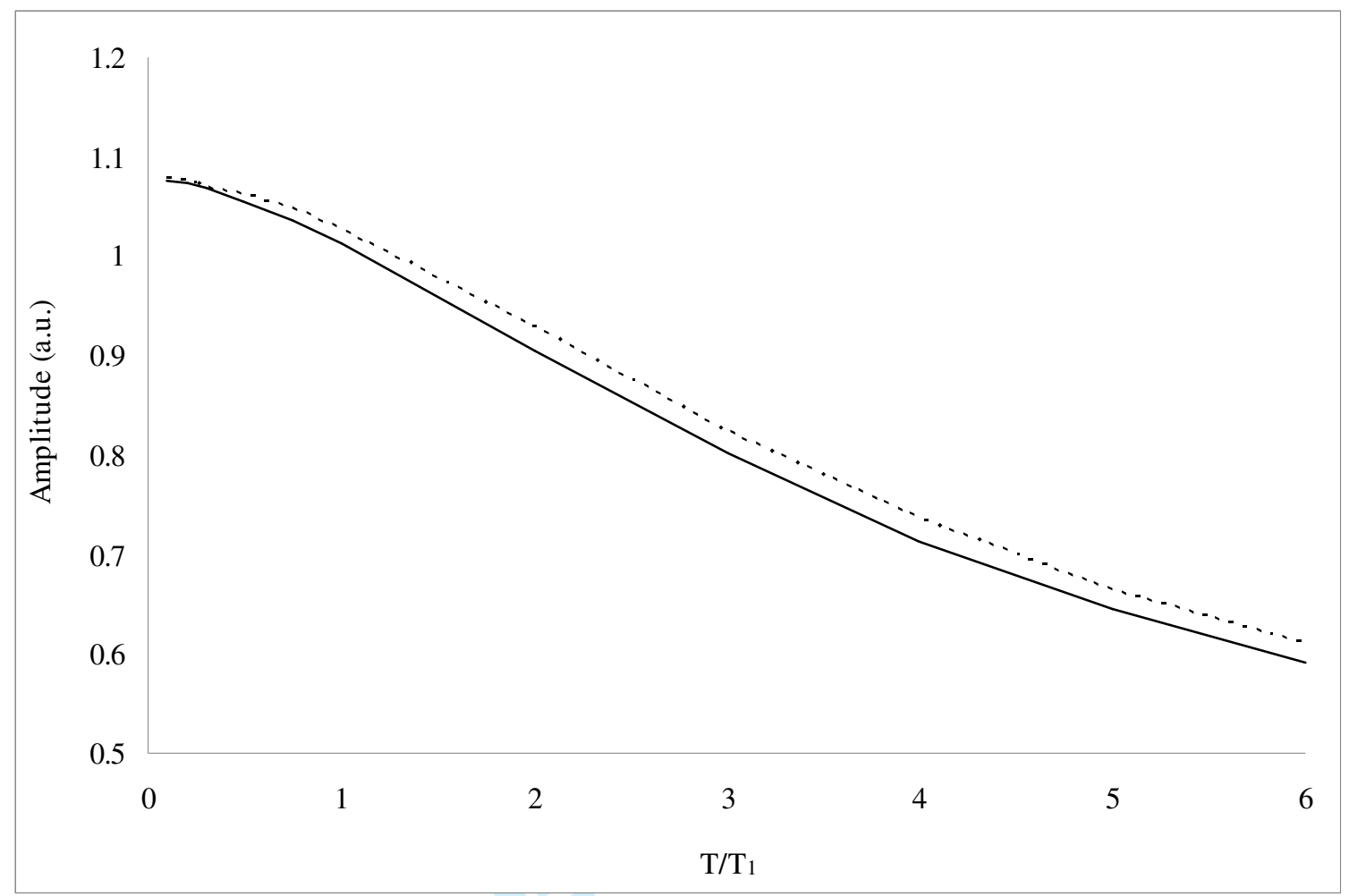

Figure 5 


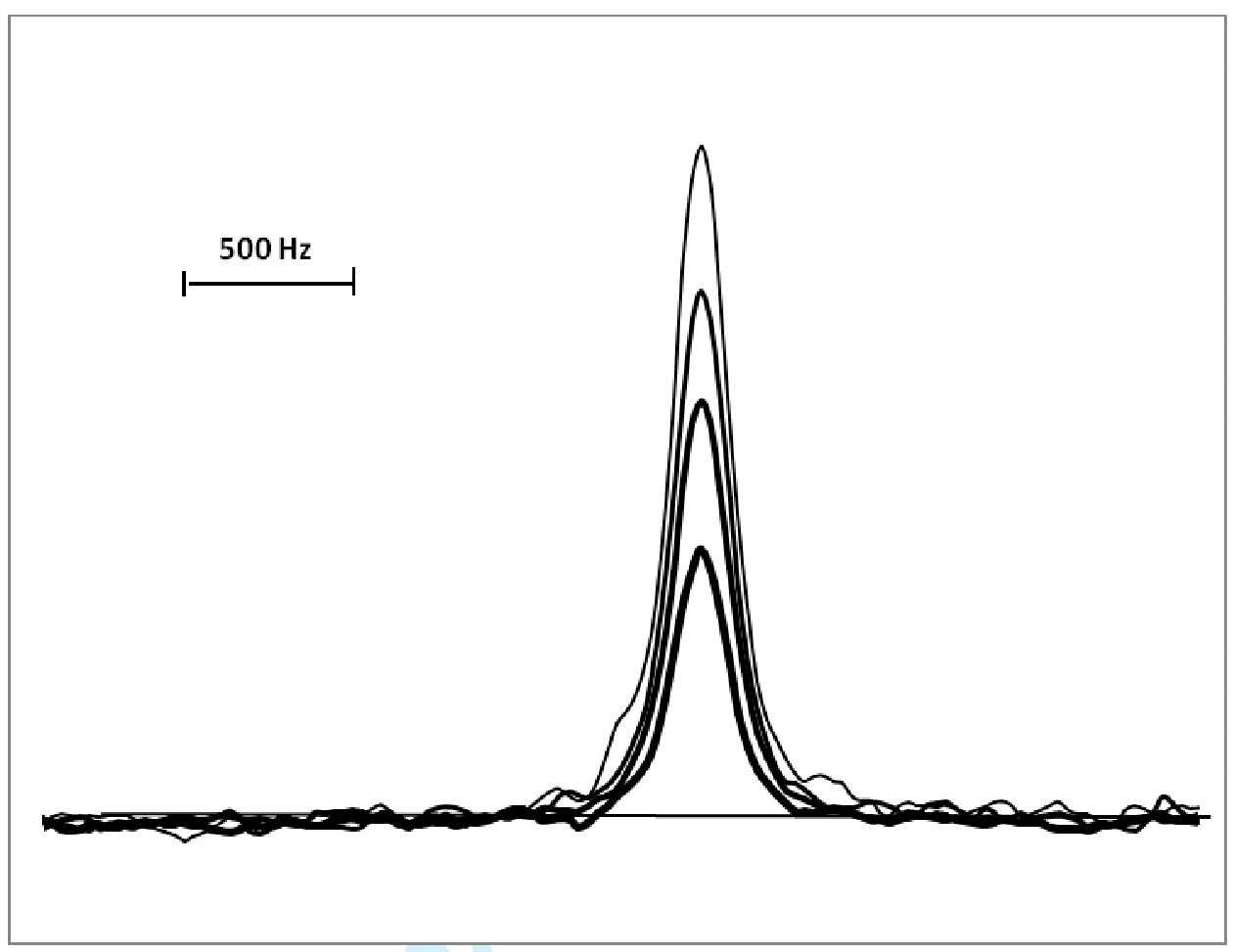

Figure 6 


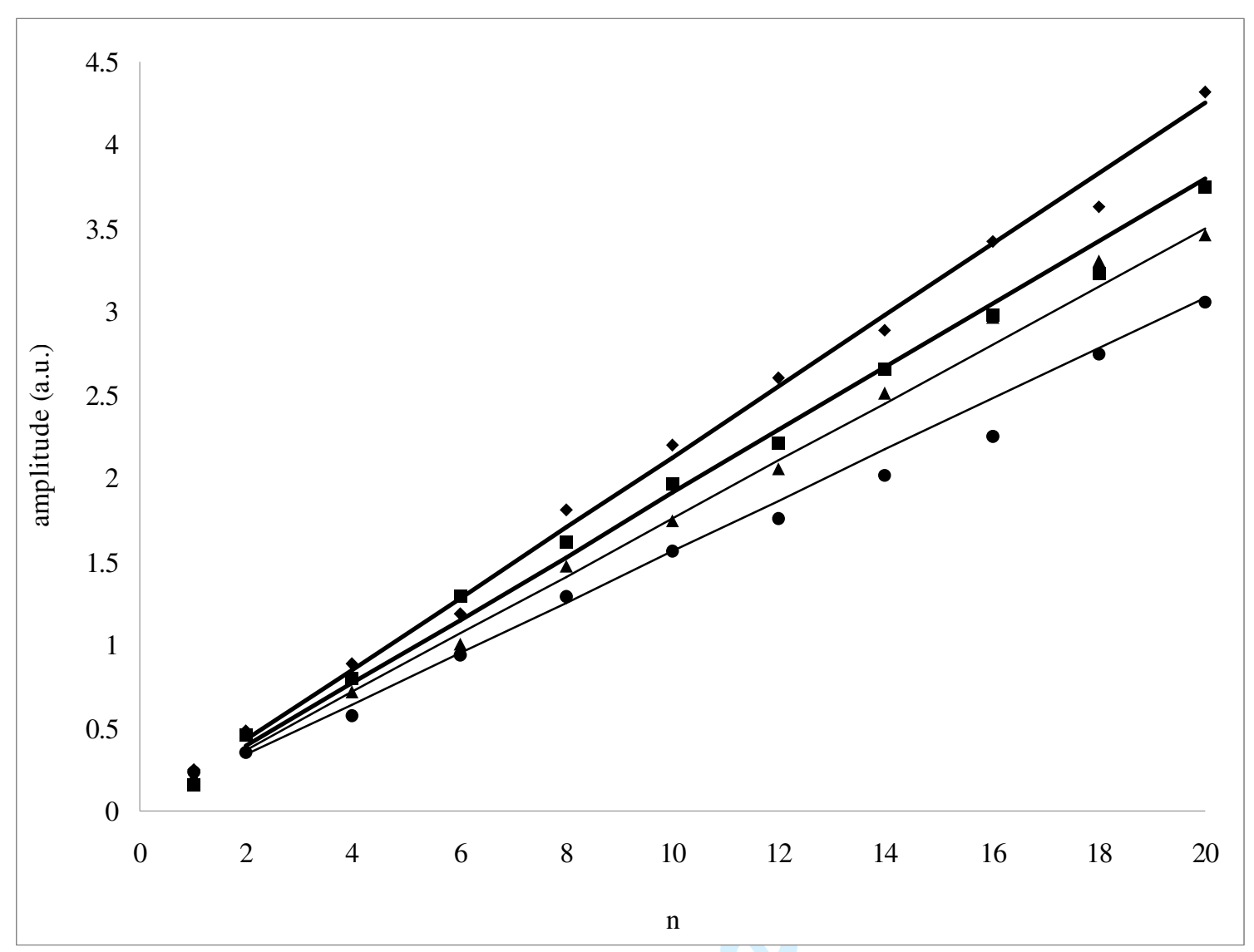

Figure 7 